\title{
Urban Stormwater Modeling with Local Inertial Approximation Form of Shallow Water Equations: A Comparative Study
}

\author{
Weiqi Wang $^{1} \cdot$ Wenjie Chen ${ }^{1} \cdot$ Guoru Huang ${ }^{1,2,3}$
}

Accepted: 26 September 2021 / Published online: 18 October 2021

(C) The Author(s) 2021

\begin{abstract}
This study focused on the performance and limitations of the local inertial approximation form model (LIM) of the shallow water equations (SWEs) when applied in urban flood modeling. A numerical scheme of the LIM equations was created using finite volume method with a first-order spatiotemporal Roe Riemann solver. A simplified urban stormwater model (SUSM) considering surface and underground dual drainage system was constructed based on LIM and the US Environmental Protection Agency Storm Water Management Model. Moreover, a complete urban stormwater model (USM) based on the SWEs with the same solution algorithm was used as the evaluation benchmark. Numerical results of the SUSM and USM in a highly urbanized area under four rainfall return periods were analyzed and compared. The results reveal that the performance of the SUSM is highly consistent with that of the USM but with an improvement in computational efficiency of approximately 140\%. In terms of the accuracy of the model, the SUSM slightly underestimates the water depth and velocity and is less accurate when dealing with supercritical flow in urban stormwater flood modeling. Overall, the SUSM can produce comparable results to USM with higher computational efficiency, which provides
\end{abstract}

Guoru Huang

huanggr@scut.edu.cn

1 School of Civil Engineering and Transportation, South China University of Technology, Guangzhou 510640, China

2 State Key Laboratory of Subtropical Building Science, School of Civil Engineering and Transportation, South China University of Technology, Guangzhou 510640, China

3 Guangdong Engineering Technology Research Center of Safety and Greenization for Water Conservancy Project, Guangzhou 510640, China a simplified and alternative method for urban flood modeling.

Keywords Comparative study · Finite volume method - Local inertial approximation form - Shallow water equations - Urban stormwater model

\section{Introduction}

Against the background of climate change and rapid urbanization that result in more frequent extreme rainfall events and changes in the underlying surface, urban floods have become a widely distributed natural hazard worldwide (Kaspersen et al. 2017). Urban floods not only impose serious security risks to human life, property, and the ecological environment, but also severely restrict the development of cities (Santos and Reis 2018).

Fast and accurate flood forecasting can provide timely hazard information for the decision makers of relevant local government departments to formulate optimal riskavoidance strategies (Carsell et al. 2004; McCallum et al. 2016). As the most important component of urban flood forecasting systems, numerical urban flood simulation technologies have attracted widespread attention in both academic research and engineering application in recent years (Liang et al. 2015; Chen et al. 2018) because of their excellent computational performance.

The urban drainage system is mainly divided into two parts, namely the surface drainage path composed of the urban road paths and river channels, and the underground pipe network system (Leandro et al. 2009). In order to simulate urban flooding in a realistic manner, urban flood models need to couple the two parts in what is referred to as the dual drainage concept. Although some studies have 
empirically generalized the conveying effect of the underground drainage system as a surface infiltration process and achieved satisfactory simulation results, an accurate urban stormwater model requires the consideration of both parts of the drainage system from the perspective of the real physical process.

The one-dimensional (1D) pipe drainage network plays an important role in the modeling of urban dual drainage systems. Many 1D sewer software packages have been extensively developed and widely used, including the Simulation of Interaction between Pipe flow and Surface Overland flow in Networks (SIPSON) package and the US Environmental Protection Agency (EPA) Storm Water Management Model (SWMM) (Gironás et al. 2010). Due to its powerful functions and robust algorithms, the SWMM, a set of open-source codes, has become the most widely used 1D hydraulic model in the world. In addition, as a distributed hydrological model, the SWMM also provides a powerful rainfall-runoff calculation capability. Therefore, it has been widely used in various urban flood studies (Yang et al. 2020; Chen et al. 2021), and has become the computational core of 1D pipe models in many commercial software, such as Infoworks-ICM, PCSWMM, and XP-SWMM.

In the past decades, regarding the solution of two-dimensional (2D) shallow water equations (SWEs), great progress has been made in terms of the development of both numerical discretization schemes and robust algorithms to address the key challenges encountered in flood modeling, such as wet/dry fronts (Castro et al. 2005), Riemann problem (Roe 1981), and sheet flow over steep slope source terms and stiff source terms (Audusse et al. 2004; Xia et al. 2017; Xia and Liang 2018). Nevertheless, there exist prevailing challenges, especially in terms of computational efficiency, which is relevant for real-time urban flood forecasting. Although GPU parallel computing technology can be used to reduce the computational time cost (Xia et al. 2019; Dazzi et al. 2020), several simplified forms of the complete SWEs, which has accuracy similar to that of the SWEs but with a less intensive flux calculation process, have been proposed, such as the diffusive wave model (DWM) and the kinematic wave model (Ponce 1990; Apel et al. 2009). As a simplified form of the complete SWEs, the local inertial approximation form model (LIM) proposed by Bates et al. (2010), which was derived by neglecting the convective acceleration term in the momentum equation, has attracted the interest of other researchers (Neal et al. 2012; de Almeida and Bates 2013; Martins et al. 2015). Via UK Environment Agency (EA) model benchmark tests, Neal et al. (2012) found that the LIM was often the most efficient model of the three main computational engines (the DWM, LIM, and full SWEs) of the LISFLOOD-FP model, and achieved good accuracy in most of the test cases. Furthermore, de Almeida and Bates (2013) pointed out that in a range of floodplain and lowland channels, the performance of the LIM becomes increasingly relevant with the increase of the Froude number and depth gradients. However, these studies were based on the finite difference method with structured grids, and were aimed at the flood characteristics of floodplains. Comparatively few studies have analyzed the performance of the LIM in urban stormwater simulation, especially in urban stormwater modeling considering a dual drainage system.

The real physical process of stormwater or flood flow in an urban dual drainage system is complex; it includes overland flow, drainage pipe flow, and river channel flow, and involves the complex flow exchange mechanism between the surface, the underground drainage pipe network, and river channels. Therefore, the coupling process of the two drainage systems has become a popular research topic. In early research, the exchange between water flow in the pipe network and the surface was considered unidirectional, and the water could only overflow from the pipe network to the surface (Hsu et al. 2000). Chen et al. (2005) were the first to overcome the difficulty of water flow exchange between the surface and underground pipe network, and truly realized the bi-directional interaction of 1D pipe flow and 2D surface flow in the modeling. Based on these studies, numerical models and physical experiments have successively emerged to carry out more detailed research on the physical mechanisms of the water exchange, coupling linkages, and synchronization schemes of the two models (Chen et al. 2007; Leandro et al. 2014).

From these perspectives, this study constructed an urban stormwater model based on the finite volume numerical scheme of the LIM, which considers the dual drainage system via coupling with the rainfall generation module and 1D pipe network module in the SWMM. The simplified urban stormwater model (SUSM) is compared with the complete urban stormwater model (USM) based on the complete SWEs in a highly urbanized area to investigate its performance and limitations in urban stormwater flood modeling.

The remainder of this article is organized as follows. Section 2 describes the numerical algorithms and the research region, and the proposed LIM is validated with test cases. Section 3 analyzes and discusses the comparison of the model results. Finally, the conclusions of this study are summarized in Sect. 4.

\section{Methodology}

In this section, the numerical schemes applied for the LIM are described and a brief introduction to the theory of the SWMM is provided. Further, the methods used for model 
coupling are introduced. Finally, an overview of the information of the research area and the data used for model setup is included.

\subsection{Overland Flow Model}

\subsubsection{Numerical Schemes}

The LIM used in this study was derived from the 2D SWEs. The SWE system consists of a mass conservation equation and momentum conservation equations in two horizontal dimensions. The mass conservation equation is as follows.

$\frac{\partial}{\partial t} h+\frac{\partial}{\partial x} h u+\frac{\partial}{\partial y} h v=0$

The momentum conservation equations are as follows.

$$
\begin{aligned}
& \frac{\partial}{\partial t} u h+\frac{\partial}{\partial x} h u^{2}+\frac{\partial}{\partial y} h u v+\frac{1}{2} \frac{\partial}{\partial x} g h^{2}+g h \frac{\partial}{\partial x} B(x, y)+\tau_{b x} \\
& \quad=0
\end{aligned}
$$

$$
\begin{aligned}
& \frac{\partial}{\partial t} v h+\frac{\partial}{\partial y} h v^{2}+\frac{\partial}{\partial x} h u v+\frac{1}{2} \frac{\partial}{\partial y} g h^{2}+g h \frac{\partial}{\partial y} B(x, y)+\tau_{b y} \\
& \quad=0
\end{aligned}
$$

where $h$ represents the water depth, $u$ and $v$ are the flow velocities in the $x$ - and $y$-directions, respectively, $g$ is the gravitational acceleration, $B(x, y)$ is the bed elevation, and $\tau_{b x}$ and $\tau_{b y}$ are respectively the bed friction stresses along the two axes.

Based on the assumption that the convective acceleration term is negligible relative to the other terms in the momentum equation, this term can be removed from the momentum equation. The LIM can then be written in the following conservative and nonlinear matrix form:

$\frac{\partial}{\partial t} \boldsymbol{U}+\frac{\partial \boldsymbol{F}}{\partial x}+\frac{\partial \boldsymbol{G}}{\partial y}=\boldsymbol{S}_{b}+\boldsymbol{S}_{f}$

with

$$
\begin{aligned}
& \boldsymbol{U}=\left[\begin{array}{c}
h \\
h u \\
h v
\end{array}\right], \boldsymbol{F}=\left[\begin{array}{c}
u h \\
\frac{1}{2} g h^{2} \\
0
\end{array}\right], \boldsymbol{G}=\left[\begin{array}{c}
u h \\
0 \\
\frac{1}{2} g h^{2}
\end{array}\right], \\
& \boldsymbol{S}_{b}=\left[\begin{array}{c}
-g h \frac{\partial}{\partial x} B(x, y) \\
-g h \frac{\partial}{\partial y} B(x, y)
\end{array}\right], \boldsymbol{S}_{f}=\frac{-g n^{2} \sqrt{u^{2}+v^{2}}}{h^{1 / 3}}\left[\begin{array}{c}
0 \\
u \\
v
\end{array}\right]
\end{aligned}
$$

where $\boldsymbol{U}$ is the vector of conserved variables, $\boldsymbol{F}$ and $\boldsymbol{G}$ are the numerical flux terms across the control volume, $\boldsymbol{S}_{b}$ and
$S_{f}$ respectively represent the slope source term and friction source term, and $n$ is the Manning roughness coefficient.

Because the finite volume method is not limited to any specific mesh structure, the discretization scheme of the governing equations can be based on an unstructured grid, which can better adapt to the complex terrain and boundary conditions in urban areas. Therefore, in this study, the LIM is solved by adopting a cell-centered finite volume method with a first-order spatiotemporal Roe scheme. For a control volume, by applying the divergence theorem to the second integral, the integral form of Eq. 4 can be expressed as

$\frac{\partial}{\partial t} \int \boldsymbol{U} d \boldsymbol{\Omega}+\oint_{\partial \Omega}(\boldsymbol{E} \cdot \overrightarrow{\boldsymbol{n}}) d s=\int \boldsymbol{S} d \boldsymbol{\Omega}$

where $\Omega$ represents the control volume, $\overrightarrow{\boldsymbol{n}}$ is the unit outward normal vector, $\boldsymbol{E}=(\boldsymbol{F}, \boldsymbol{G})^{T}$, and $\boldsymbol{S}=\left(\boldsymbol{S}_{b}, \boldsymbol{S}_{f}\right)^{T}$.

By applying the discrete approximation scheme to each control cell $i$, the following is obtained:

$\frac{\partial \boldsymbol{U}_{i}}{\partial t} A_{i}+\oint_{\partial \Omega}(\boldsymbol{E} \cdot \overrightarrow{\boldsymbol{n}}) d s=\int \boldsymbol{S} d \Omega$

where the subscript $i$ indicates the index number of the control volume, and $A_{i}$ is the area of control cell $i$.

For the flux term, a numerical solution scheme based on the Riemann solver presented by Brufau et al. (2002) is adopted. A local Riemannian problem is defined according to the state of the conserved variables on the left and right sides of each cell edge. Assuming that the numerical flux is at the midpoint of each cell edge,

$\oint_{\partial \Omega}(\boldsymbol{E} \cdot \overrightarrow{\boldsymbol{n}}) d s \approx \sum_{K=1}^{N E}\left(\left(\boldsymbol{E}_{\boldsymbol{k}} \cdot \overrightarrow{\boldsymbol{n}}_{\boldsymbol{k}}\right) d l_{k}\right)$

where $N E$ represents the number of edges of a control cell, the subscript $K$ represents the edge index of each cell, and $l_{k}$ is the length of the $\mathrm{k}$-th edge.

The Jacobian matrix $\mathbf{J}_{R L}$ of the normal numerical flux for the LIM can be derived as

$\mathbf{J}_{R L}=\left(\begin{array}{ccc}0 & \overrightarrow{\boldsymbol{n}}_{x} & \overrightarrow{\boldsymbol{n}}_{y} \\ c^{2} \overrightarrow{\boldsymbol{n}}_{x} & 0 & 0 \\ c^{2} \overrightarrow{\boldsymbol{n}}_{y} & 0 & 0\end{array}\right)$

with the following eigenvalues and the corresponding eigenvectors:

$\boldsymbol{e}^{1}=\left(\begin{array}{c}1 \\ c \overrightarrow{\boldsymbol{n}}_{x} \\ c \overrightarrow{\boldsymbol{n}}_{y}\end{array}\right), \boldsymbol{e}^{2}=\left(\begin{array}{c}0 \\ -c \overrightarrow{\boldsymbol{n}}_{y} \\ -c \overrightarrow{\boldsymbol{n}}_{x}\end{array}\right), \boldsymbol{e}^{3}=\left(\begin{array}{c}1 \\ -c \overrightarrow{\boldsymbol{n}}_{x} \\ -c \overrightarrow{\boldsymbol{n}}_{y}\end{array}\right)$

$\lambda^{1}=c, \lambda^{2}=0, \lambda^{3}=-c$

where $\boldsymbol{e}$ and $\lambda$ respectively represent the eigenvectors and the eigenvalues of Jacobian matrix $\mathbf{J}_{R L}$, and $c=\sqrt{g h}$ is the surface wave celerity. 
An approximate Jacobian matrix $\tilde{\mathbf{J}}_{R L}$ suggested by Roe (1981) is adopted for the 1D Riemannian problem; while it has the same shape as $\mathbf{J}_{R L}$, it is recalculated with the Roe average state given by the quantities $\boldsymbol{u}=(\tilde{u}, \tilde{v})$ and $\tilde{c}$. The numerical flux $\phi_{R L}$ across the cell edge can be expressed as

$\phi_{R L}=\frac{1}{2}\left(\boldsymbol{E}_{R}+\boldsymbol{E}_{L}\right)-\frac{1}{2}\left|\tilde{J}_{R L}\right|\left(\boldsymbol{U}_{R}-\boldsymbol{U}_{L}\right)$

where the subscripts $R$ and $L$ represent the left and right sides of the cell edge, respectively.

Considering the convenience of calculation and programming, matrix $\left|\tilde{\boldsymbol{J}}_{R L}\right|$ can be replaced by the product form of the eigenvalues and eigenvectors, as follows.

$\left|\tilde{\boldsymbol{J}}_{R L}\right|\left(\boldsymbol{U}_{R}-\boldsymbol{U}_{L}\right)=\sum_{K=1}^{N E}\left|\sim_{\lambda}^{K}\right| \alpha^{K} \underset{\sim}{\sim^{K}}$

The expressions of the coefficients $\alpha$ deduced for the LIM are as follows.

$\alpha^{1,3}=\frac{1}{2}\left(h_{R}-h_{L}\right) \pm \frac{1}{2 \tilde{c}}\left[\left(h_{R} u_{R}-h_{L} u_{L}\right) \overrightarrow{\boldsymbol{n}}_{x}+\left(h_{R} v_{R}\right.\right.$ $\left.\left.-h_{L} v_{L}\right) \overrightarrow{\boldsymbol{n}}_{y}\right]$,

$\alpha^{2}=\frac{1}{\tilde{c}}\left[\left(\left(h_{R} v_{R}-h_{L} v_{L}\right) \overrightarrow{\boldsymbol{n}}_{x}-\left(h_{R} u_{R}-h_{L} u_{L}\right) \overrightarrow{\boldsymbol{n}}_{y}\right]\right.$

Finally, the formula of the numerical flux term can be deduced as follows.

$\phi_{P Q}=\left[\begin{array}{l}\frac{1}{2}\left[\left(h_{L}-h_{R}\right) \tilde{c}+\left(h_{L} u_{L}+h_{R} u_{R}\right) \vec{n}_{x}+\left(h_{L} v_{L}+h_{R} v_{R}\right) \vec{n}_{y}\right] \\ \frac{1}{2}\left[\frac{g}{2}\left(h_{L}^{2}+h_{R}^{2}\right) \vec{n}_{x}+\tilde{c} \vec{n}_{x}^{2}\left(h_{L} u_{L}-h_{R} u_{R}\right)+\tilde{c} \vec{n}_{x} \vec{n}_{y}\left(h_{L} v_{L}-h_{R} v_{R}\right)\right] \\ \frac{1}{2}\left[\frac{g}{2}\left(h_{L}^{2}+h_{R}^{2}\right) \vec{n}_{y}+\tilde{c} \vec{n}_{y}^{2}\left(h_{L} v_{L}-h_{R} v_{R}\right)+\tilde{c} \vec{n}_{x} \vec{n}_{y}\left(h_{L} u_{L}-h_{R} u_{R}\right)\right]\end{array}\right]$

A novel surface reconstruction method (SRM) proposed by Xia et al. (2017) is adopted to solve the bed elevation source term. This numerical scheme reconstructs the water level and bed elevation on both sides of the cell interface with the slope (gradient) limiter of the bed elevation to compute the corresponding Riemann states of the flow variables. The Riemann states obtained by the SRM are then applied to calculate the numerical fluxes with the Roe Riemann solver, as mentioned previously. The friction source term is discretized with a novel implicit approach proposed by Xia and Liang (2018). With the analytic derivation of the fully implicit scheme solution, the flow variables can be explicitly updated. This novel approach effectively solves the problems caused by the relationship between gravity and friction when dealing with the friction source term. After the spatial discretization schemes are determined, the final numerical scheme for the LIM in this study can be written as
$\boldsymbol{U}^{j+1}=\boldsymbol{U}^{j}-\frac{\Delta t}{A_{i}} \sum_{k=1}^{N E} \boldsymbol{E}_{k}^{j} \overrightarrow{\boldsymbol{n}}_{\boldsymbol{k}} l_{k}+\Delta t\left(\boldsymbol{S}_{b}^{j}+\boldsymbol{S}_{f}^{j+1}\right)$

where superscript $j$ and $j+1$ respectively represent the current time step and next time step, and $\Delta t$ is the calculation time step. Benefiting from the novel scheme of the friction source term, the time step is only strictly limited by the Courant-Friedrichs-Levy (CFL) condition. As the stability criterion in this study, the CFL condition is slightly different from that of the SWEs because the LIM only includes the information of momentum (-c and c), and, as shown by Bates et al. (2010):

$\Delta t=C F L \frac{d_{i}}{\sqrt{g h}}$

where $C F L$ is a stable coefficient in the range of $0.2-0.7$, and $d_{i}$ is the minimum distance from the cell center to the edge.

\subsubsection{Model Evaluation}

Two test cases were examined to evaluate the numerical schemes for the LIM proposed in Sect. 2.1.1. The shallow water model (SWM), which is based on the complete SWEs (the numerical solution to the SWM is the same as the LIM), was constructed to demonstrate the differences between the two models and verify the convergence and accuracy of the LIM.

The Stoker dam-break test simulates the flow movement of dam-break floods. Its theoretical solution was derived by Stoker in 1957, and it has since been widely used to test the accuracy of hydrodynamic model solutions. The Stoker dam-break test assumes a Riemann problem in a channel of constant width with a flat and zero-resistance bottom. The basic settings and initial conditions are shown in Fig. 1, and all the boundary conditions in this study were solid wall boundaries. The simulation results for $t=50 \mathrm{~s}$ were used for analysis and comparison, and the corresponding theoretical profiles for the water level and velocity are also plotted as standards of comparison, as shown in Fig. 2.

Figure 2 verifies the performance of the numerical schemes used in this study. It can be seen that the propagation speed of the front wave simulated by the LIM was relatively slower than that of the SWM. This phenomenon can be explained by the characteristic theory; in the LIM, the speed of the front wave contained in the first/third characteristic fields is always negative/positive, and is different from that in the SWM. Furthermore, because the mass conservation equations of the two models are the same, the difference caused by the front wave speed also affects the water level along the channel. While the two models are slightly different, their results were still similar 
Fig. 1 The diagram of the Stoker dam-break test

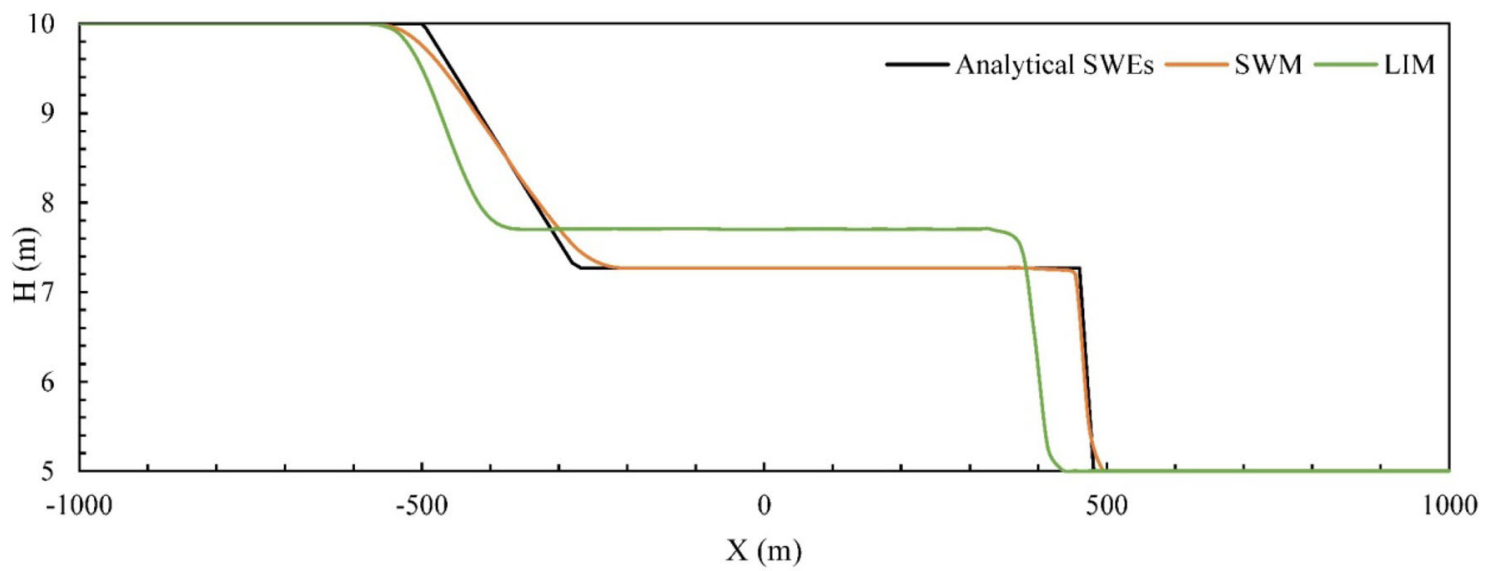

(a)

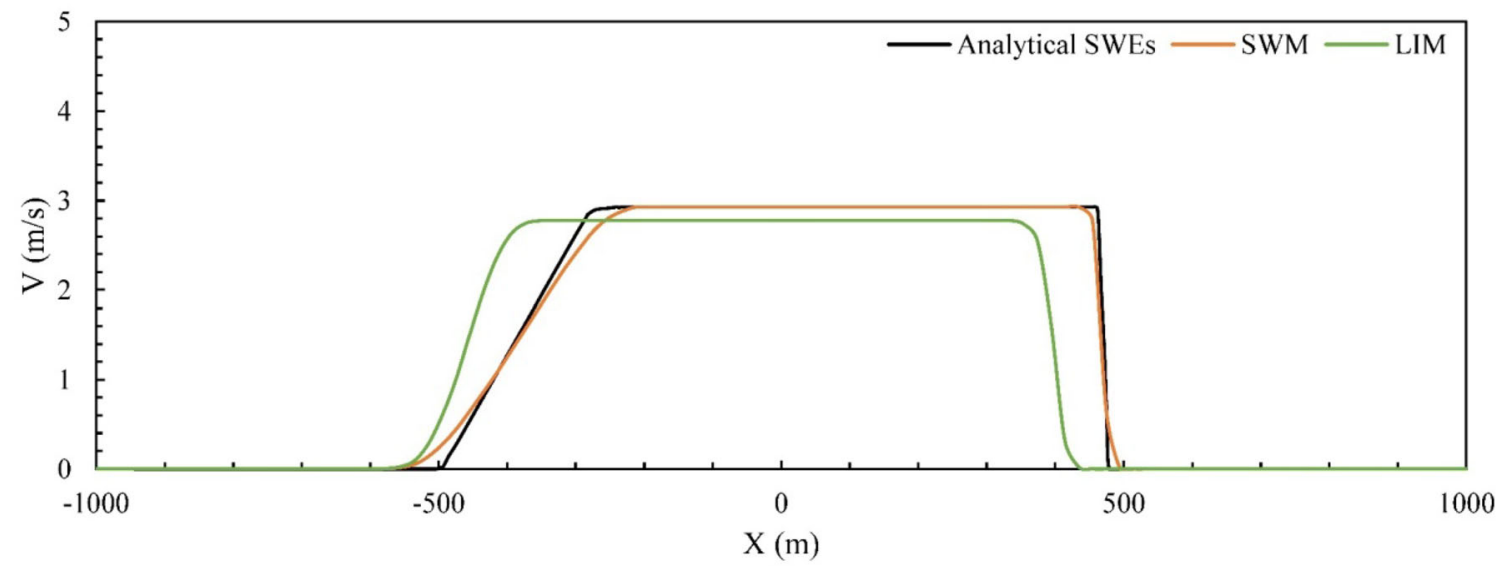

(b)

Fig. 2 Profiles of the water depth $\mathrm{H}$ ( a) and flow velocity V (b). SWM = shallow water model, LIM = local inertial approximation form model

to the theoretical solution. Furthermore, the computational efficiency of the LIM exhibited an improvement of $164 \%$ as compared to that of the SWM (LIM: $7.95 \mathrm{~s}$; SWM: $13 \mathrm{~s}$ ).

For the flooding test of a disconnected water body, a 100-m-wide, 700-m-long domain with a longitudinal profile was considered and a water level boundary condition was set at $\mathrm{x}=0 \mathrm{~m}$, as illustrated in Fig. 3. The rest of the boundaries were all solid wall boundaries. Moreover, Manning's $\mathrm{n}$ was set as 0.03 (uniform). The objective of this test was to assess the basic capabilities of the models, such as their ability to deal with disconnected water bodies and the wetting and drying of floodplains. The EA (Néelz and Pender 2013) tested the performance of the latest generation of 2D hydraulic modeling tools via a series of cases. Therefore, the results of the EA tests at two test points ( $x=400 \mathrm{~m}$ and $x=600 \mathrm{~m}$ ) based on the same case were obtained for comparison, as exhibited in Fig. 4.

It is evident that the LIM and the SWM constructed in this study both exhibited excellent performance. At point 1 , both models predicted an initial sheet flow, a similar rate of water level rise within $\mathrm{t}=1-2 \mathrm{~h}$, a maximum water level at $10.35 \mathrm{~m}$, and finally a pond water level at $10.25 \mathrm{~m}$. At point 2, the water level results of the LIM were largely the same as the results of other models. Moreover, compared to the SWM, the computational efficiency was increased by about 171\% (LIM: 166.5 s; SWM: 284.8 s).

The two benchmark tests demonstrate that the proposed solution of the LIM achieved satisfactory performance. Furthermore, benefiting from the simplification of the numerical flux term and the relatively loose CFL condition, 


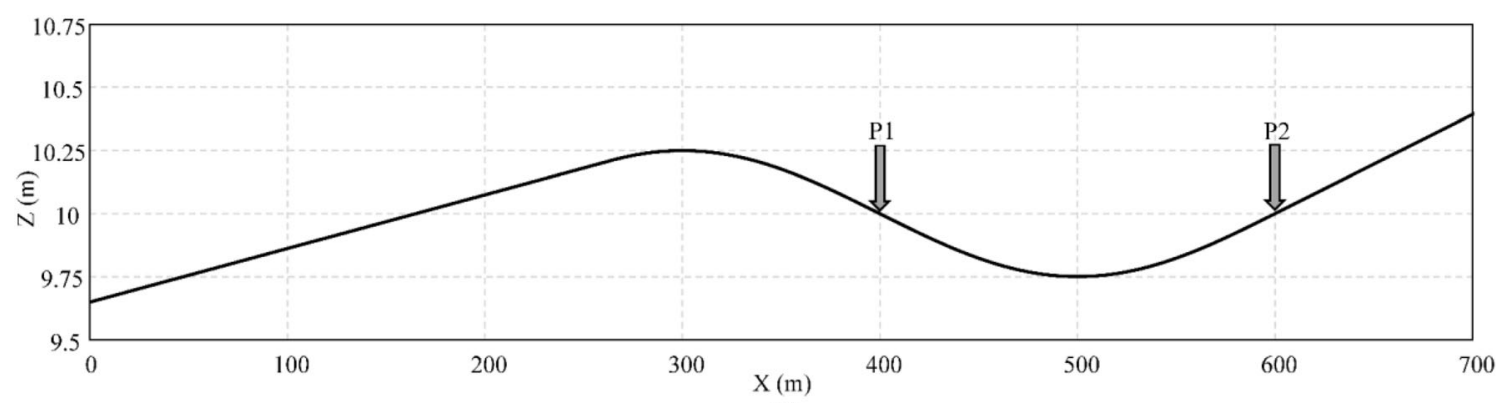

(a)

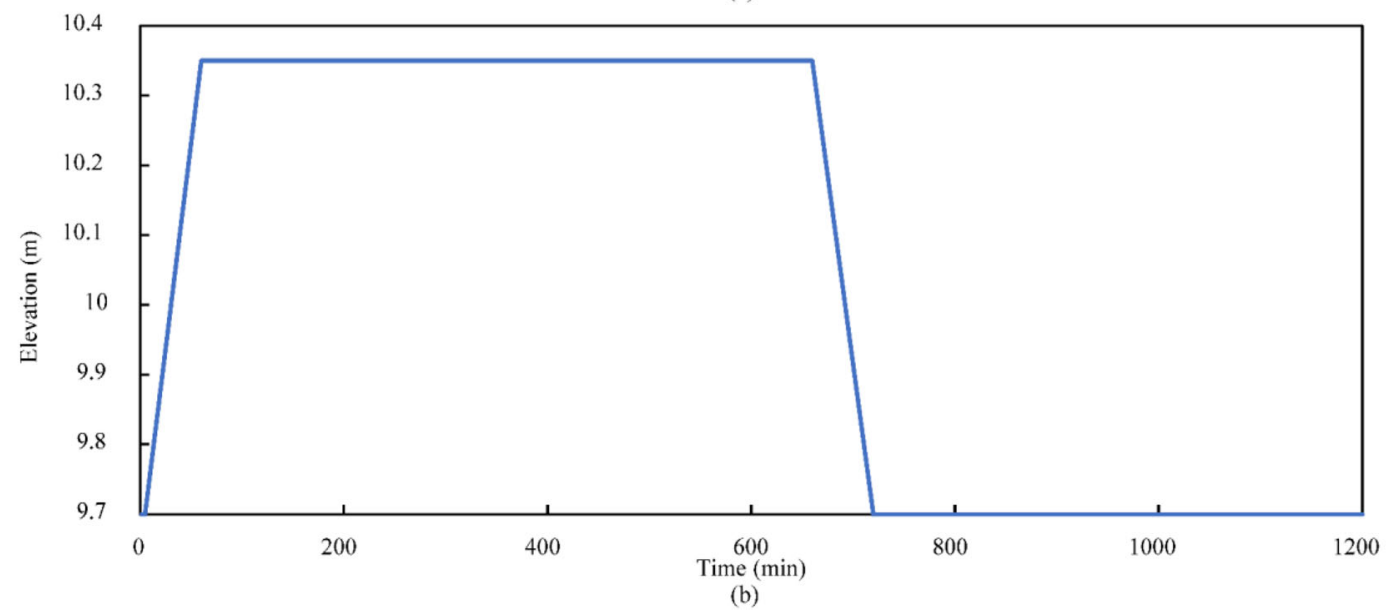

Fig. 3 Diagram of the flooding test of a disconnected water body a and the water level boundary condition at $x=0 \mathrm{~m} \mathbf{b}$

the calculation efficiency was improved by up to $164 \%-$ $171 \%$ with similar accuracy as the SWM.

\subsection{Storm Water Management Model (SWMM)}

The SWMM is a dynamic rainfall-runoff simulation program developed by the US EPA. It has been widely used in the past few decades for the single-event or long-term (continuous) simulation of the runoff and water quality in urban areas. In this research, partial program modules in the SWMM were applied to calculate the rainfall-runoff process and the conveyance portion of the drainage system (including the underground pipe network and river channels) during an urban flooding event caused by rainfall.

As a distributed model, the SWMM allows the study area to be divided into any number of sub-catchment areas with irregular shapes. In the SWMM, the surface runoff generated by rainfall in the study area is calculated based on each sub-catchment using a nonlinear reservoir model (Chen and Shubinski 1971). The movement of water flow in the pipe/channel drainage system is controlled by the mass and momentum conservation equations of gradually changing unsteady free surface flow, and node continuity equation is added, as the drainage system in the SWMM is mainly composed of pipes and nodes. The SWMM solves the governing equations using the implicit finite difference method with successive approximation, and more detailed solution schemes to the governing equations are introduced in Rossman and Huber (2017).

\subsection{Model Coupling}

This study simultaneously simulated the flow dynamics in sewer networks, rivers, and on overland surfaces by coupling the SWMM and LIM. Three types of linkages, including vertical, lateral, and longitudinal linkages, were implemented and studied here.

\subsubsection{Three Types of Linkages}

The flow dynamics in a pipe/channel drainage system and on the urban surface were respectively calculated by the SWMM and LIM. The water exchange between the 1D drainage network and 2D surface is the key factor for the construction of a coupled 1D-2D urban stormwater model.

In this study, the two models were executed independently in the same time step. At the end of each time step, the water exchange information between the junction node and surface computing cell was calculated in consideration of three types of appropriate linkages (Chen et al. 2007), namely vertical, lateral, and longitudinal linkages. These three types of linkages are the main ways to realize the 

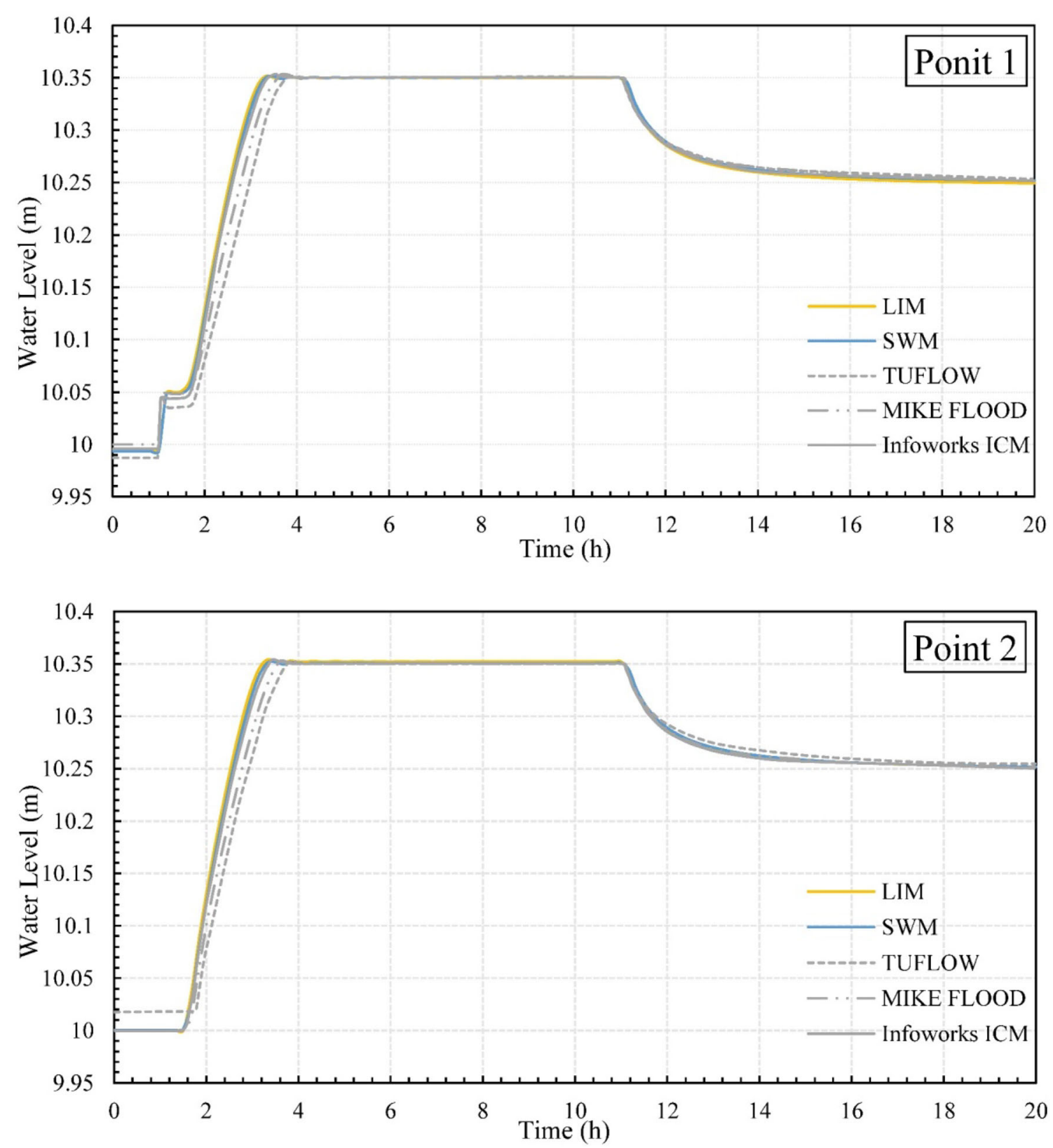

Fig. 4 Comparison of the water level at points 1 and 2 for the flooding test of a disconnected water body based on the local inertial approximation form model (LIM), shallow water model (SWM), and business software results

exchange of water flow between pipe networks/river channels/reservoirs and overland surface flow. The weir flow formula and orifice flow formula are used to calculate the flow exchange $Q_{e x}$.

For vertical linkages,

$Q_{e x}=\left\{\begin{array}{l}c_{w} w h_{2 D} \sqrt{2 g h_{2 D}} \\ c_{o} w A_{m h} \sqrt{2 g\left(H_{2 D}-H_{1 D}\right)}\end{array}\right.$

if $H_{1 D} \leq Z_{2 D}<H_{2 D}$

if $Z_{2 D} \leq H_{1 D}<H_{2 D}$

where $c_{w}$ and $c_{o}$ respectively represent the weir flow coefficient and the orifice flow coefficient, $w$ and $A_{m h}$ respectively denote the perimeter and area of the manhole, $H_{2 D}$ is the water level of the surface cell, $H_{1 D}$ is the pressure head of the manhole, and $Z_{2 D}$ is the bed elevation.

For lateral linkages,

$Q_{e x}= \begin{cases}0.35 b_{e} h_{\max } \sqrt{2 g h_{\max }} & \text { if } \frac{h_{\min }}{h_{\max }} \leq \frac{2}{3} \\ 0.91 b_{e} h_{\min } \sqrt{2 g\left(h_{\max }-h_{\min }\right)} & \text { if } \frac{2}{3}<\frac{h_{\min }}{h_{\max }} \leq 1\end{cases}$ 
with $h_{\max }=\max \left(H_{1 D}, H_{2 D}\right)-Z_{w}$ and $h_{\min }=\min \left(H_{1 D}\right.$, $\left.H_{2 D}\right)-Z_{w}$, where $b_{e}$ is the width of the weir and $Z_{w}$ is the elevation of the weir.

For these two linkages, the exchange discharge calculated by the formulas will be deducted from or added to the overland surface flow system via the operation of the source term of the LIM. Furthermore, a corresponding deduction or addition in the 1D flow system will be made via the modification of the node discharge in the SWMM.

For longitudinal linkages, the SWMM extracts the water level data of the linked 2D mesh as the boundary condition of the next time step, and provides the flow rate to the linked 2D mesh as its boundary condition using the following equations:

$H_{1 D}=\sum_{k=1}^{M} \frac{Z_{k} l_{k}}{L}, Q_{1 D}=\sum_{k=1}^{M} q_{k} l_{k}$

where $H_{1 D}$ is the water level condition for the SWMM, $Q_{1 D}$ is the discharge condition for the LIM, $Z_{k}$ and $q_{k}$ respectively represent the water level and flow rate of the linked cell edge $k$, and $M$ is the number of linked cells.

\subsubsection{Coupling Implementation}

The SWMM (version 5.1 was used in this study) source code is based on $\mathrm{C}$ language development, and provides a dynamic link library (DLL) for researchers to carry out related secondary development. Furthermore, based on the LIM algorithms proposed in this article, the construction of the 2D urban surface flow numerical model was realized with the $\mathrm{C} / \mathrm{C}++$ language. Some additional related functions were extended to DLL codes to realize the bidirectional dynamic coupling of the two models, even though the original DLL code contains several interface functions for external calls.

\subsection{Research Region}

The Minzhi region located in Shenzhen City, Guangdong Province, China, was selected as the research region (Fig. 5). The region is typically characterized by intensive urbanization with a highly concentrated population and property. The Minzhi region is located in the south of the Tropic of Cancer, and has a subtropical marine climate. There is abundant rainfall in the basin, with an average annual rainfall of $1,822 \mathrm{~mm}$. The annual rainfall is mainly concentrated from April to September, which accounts for $84 \%$ of the total. In recent years, extreme rainfall events have occurred frequently in the region, which seriously affected people's lives and the economy, as well as the natural environment. Based on the analysis of the drainage network service scope and topographical conditions, a
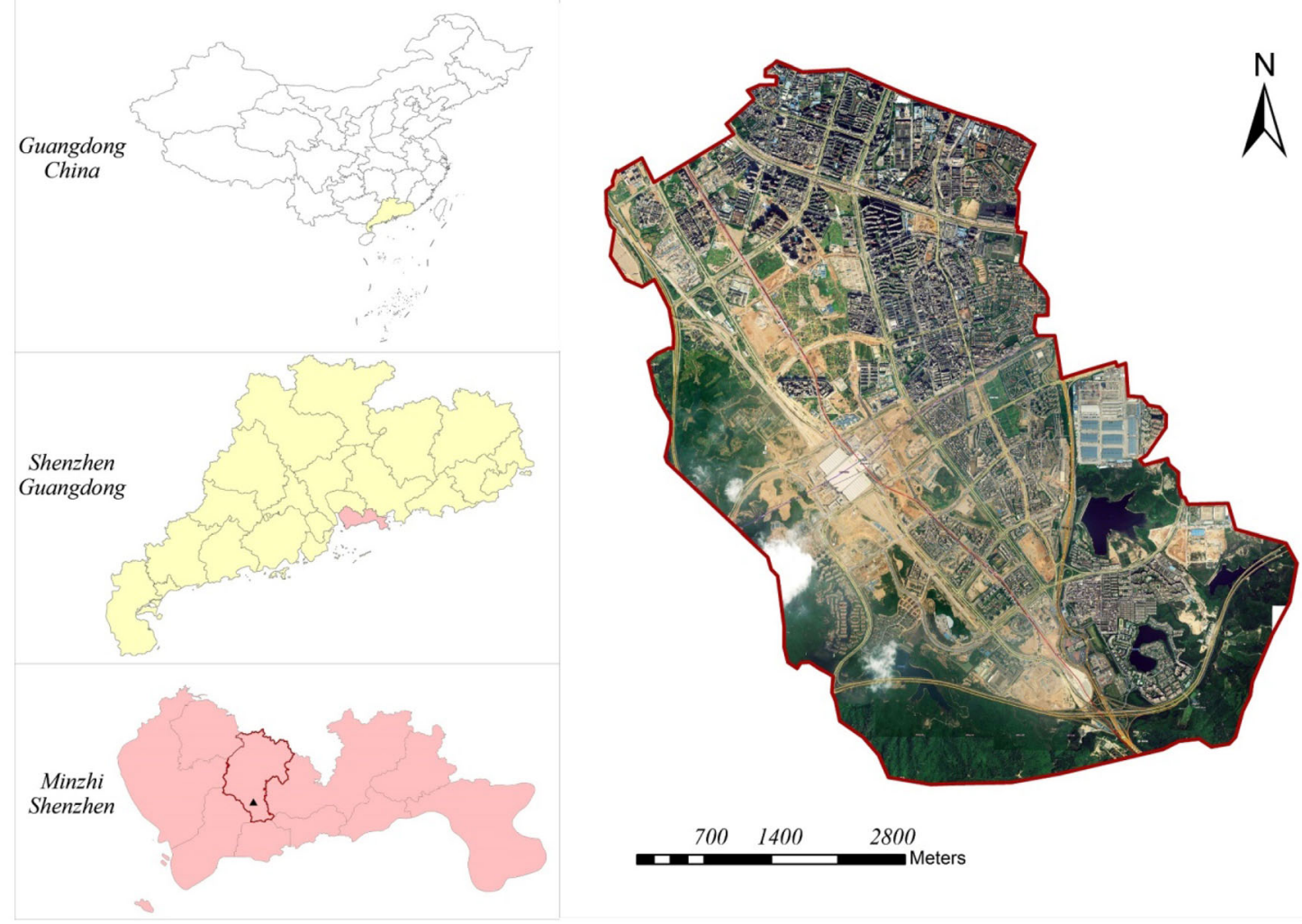

Fig. 5 Location of the study area 
Fig. 6 Hydrographs of the four rainfall return periods (10a, 20a, $50 \mathrm{a}$, and 100a)

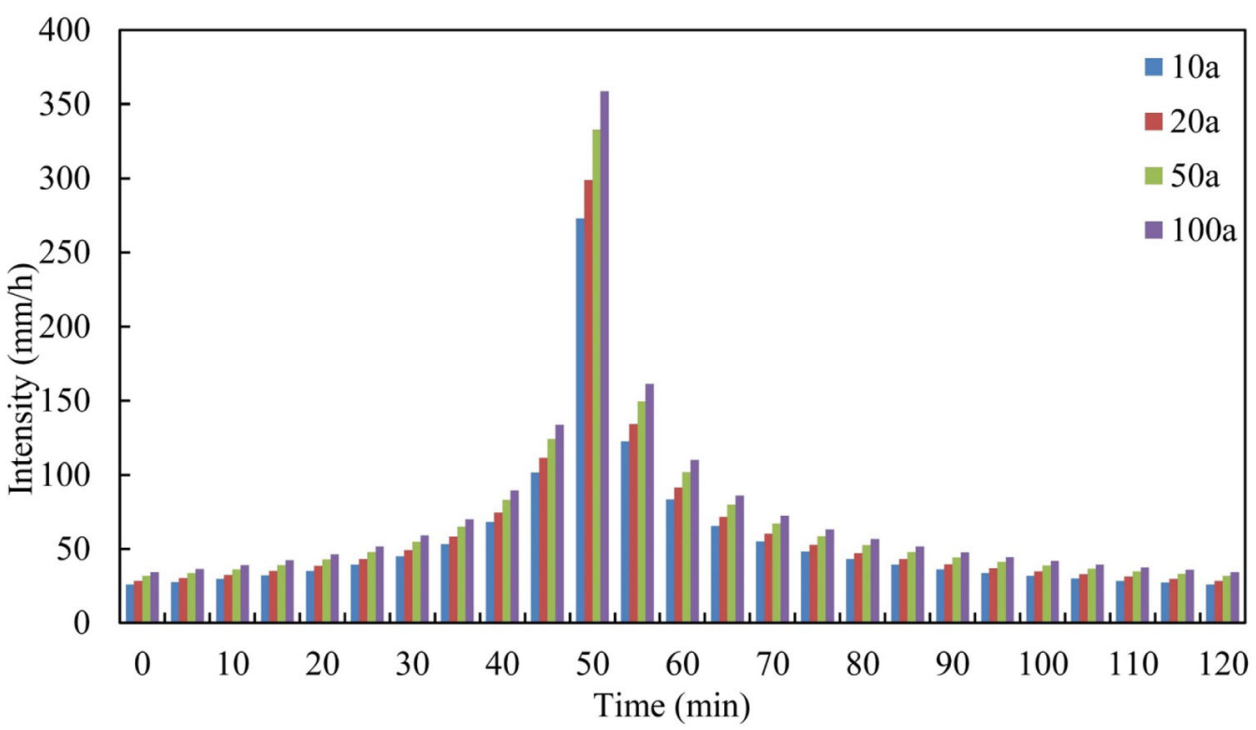

closed watershed in Minzhi with an area of $25.33 \mathrm{~km}^{2}$ was selected as the study area.

\subsection{Model Setup}

The digital elevation model (DEM) of the study area was derived via the interpolation of 1:2,000 scattered highdensity elevation points. Furthermore, the build-hole method was adopted to reflect the impermeability of buildings. The contour of impervious buildings identified via high-resolution Google remote sensing images was used as the boundary of the non-submerged area.

Drainage network data obtained from the Shenzhen Water Affairs Bureau were used to construct the drainage system model. The Minzhi River in the study area was regarded as rectangular open channels in the SWMM. Moreover, there were 2,968 junction nodes, 25 outfall nodes, and 5,051 conduit links in the model.

A recorded historical rainfall event was used to verify the coupled model, and the rainfall data were obtained from the China Meteorological Data Service Center. ${ }^{1}$ Reliable inundation information is often the greatest obstacle to the verification of urban stormwater models, as it is difficult to obtain the detailed submergence range and timely data during a rainfall event. The most commonly used method to verify the reliability of urban stormwater models is via the maximum water depth data of some locations in the city. According to the investigation of waterlogging during this rainfall event, the Shenzhen Water Bureau provided the maximum submerged depth in several locations as the standard for model validation.

In addition, four rainfall return periods of $10 \mathrm{a}, 20 \mathrm{a}, 50 \mathrm{a}$, and $100 \mathrm{a}$, were adopted to examine the performance of the

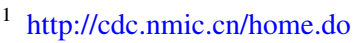

proposed urban stormwater model (Fig. 6). According to the Bureau of Municipal and Rural Construction of Shenzhen City, the rainfall intensity in Shenzhen can be summarized using the following equation:

$q=\frac{1535.398 \times(1+0.46 \lg P)}{(t+6.84)^{0.555}}$

where $q$ is the rainfall intensity, $P$ is the rainfall return period and $t$ is the rainfall duration. The Chicago approach was used to redistribute the rainfall amounts before and after the peaks. The rainfall duration was set as $120 \mathrm{~min}$, and the recording interval was $5 \mathrm{~min}$.

\section{Results and Discussion}

This section presents the most important outcomes of our research on the performance and limitations of the SUSM when applied in urban flood modeling.

\subsection{Urban Stormwater Model Validation}

Considering that the complete SWEs have a more accurate physical meaning, the validation of the models was based on the USM. Most of the parameters that need to be calibrated are in the SWMM. The key parameters for the coupled model are the Manning coefficient, depression storage depth, and Horton infiltration parameters. Thus, these parameters are calibrated within reasonable limits by comparing model results with recorded data. Table 1 shows the key parameters in the coupled model. Furthermore, to reasonably analyze and compare the two models, the simulation results of both models reported in the subsequent subsections were obtained based on the same 
parameters. Figure 7 displays the modeled inundation range and maximum depth, as well as the measured urban waterlogging data of the study area during the historical rainfall event. Table 2 reports the detailed comparison

Table 1 The key parameters for the coupled model

\begin{tabular}{llll}
\hline Parameter & Type & Value & Unit \\
\hline Manning coefficient & Pipe & 0.013 & $\mathrm{~s} / \mathrm{m}^{1 / 3}$ \\
& Pervious area & 0.24 & $\mathrm{~s} / \mathrm{m}^{1 / 3}$ \\
& Impervious area & 0.011 & $\mathrm{~s} / \mathrm{m}^{1 / 3}$ \\
Depression storage depth & Pervious area & 5 & $\mathrm{~mm}$ \\
& Impervious area & 2.5 & $\mathrm{~mm}$ \\
Horton infiltration parameters & Maximum rate & 78.1 & $\mathrm{~mm} / \mathrm{h}$ \\
& Minimum rate & 3.3 & $\mathrm{~mm} / \mathrm{h}$ \\
& Decay constant & 3.35 & $1 / \mathrm{h}$ \\
& Drying time & 6 & day \\
\hline
\end{tabular}

between the simulated results and the measured data from the study area with detailed water depth information.

The validation results reveal that the urban stormwater model correctly matched the recorded data over a large area, and the errors between the simulation results and the measured data were minor. Thus, the model constructed for the Minzhi region has considerable reliability for the simulation of stormwater inundation.

Table 2 Validation of the simulation results and the measured data

\begin{tabular}{llll}
\hline Location & Measured $(\mathrm{m})$ & Simulated $(\mathrm{m})$ & Error $(\mathrm{m})$ \\
\hline P1 & 1.3 & 1.168 & -0.132 \\
P2 & 0.6 & 0.683 & 0.083 \\
P3 & 0.4 & 0.352 & -0.048 \\
P4 & 0.6 & 0.508 & -0.092 \\
\hline
\end{tabular}

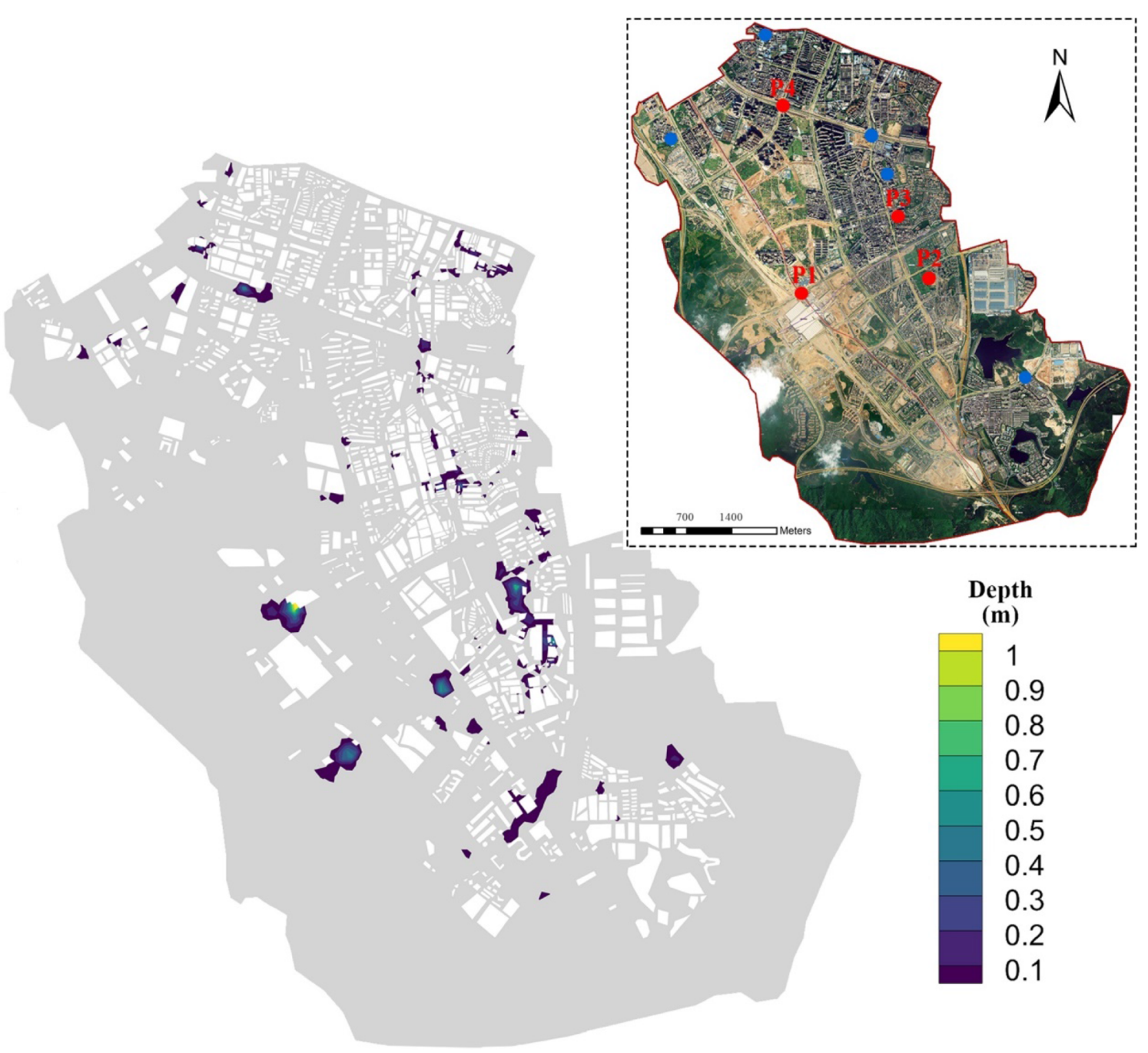

Fig. 7 Simulation results and the measured data from the study area (red dots: area with water depth data; blue dots: area without water depth data) 


\subsection{Computational Efficiency}

Table 3 reports the detailed comparison of the computational time and efficiency of the two models for the four return periods. With the increase of the rainfall return period, the larger number of wet cells involved in the flux calculation in the study area led to the increase of the calculation time of the models. Further, the analysis

Table 3 Comparison of the two models in terms of the computational time cost (CPU time)

\begin{tabular}{lllll}
\hline Return Period & $10 \mathrm{a}$ & $20 \mathrm{a}$ & $50 \mathrm{a}$ & $100 \mathrm{a}$ \\
\hline USM & $240.13 \mathrm{~s}$ & $256.62 \mathrm{~s}$ & $265.58 \mathrm{~s}$ & $270.5 \mathrm{~s}$ \\
SUSM & $174.82 \mathrm{~s}$ & $180.40 \mathrm{~s}$ & $182.15 \mathrm{~s}$ & $183.69 \mathrm{~s}$ \\
Improvement & $137 \%$ & $142 \%$ & $145 \%$ & $147 \%$ \\
\hline
\end{tabular}

USM = urban stormwater model; SUSM $=$ simplified urban stormwater model reported in Sect. 2.1 indicated that the simplification of the flux computation due to the neglect of the convective acceleration term and the inconsistency of the time steps calculated by the different CFL condition led to an enhancement in the computational efficiency of the LIM by $164 \%-171 \%$. The efficiency comparison demonstrates that this feature is still reflected in the coupled urban stormwater model. However, due to the calculation process of the pipe network and model coupling, which constituted a large part of the entire numerical simulation, the increase in efficiency was reduced to approximately $140 \%$; nonetheless, it still reflects a satisfactory improvement.

\subsection{Water Volume}

As key and direct indicators of flood inundation, the surface water volume and pipe network water volume were selected for model analysis and comparison. Figure 8
Fig. 8 Water volume hydrographs of the local inertial approximation form model (LIM) and Storm Water Management Model (SWMM) in the four rainfall return periods. USM = urban stormwater model; SUSM = simplified urban stormwater model
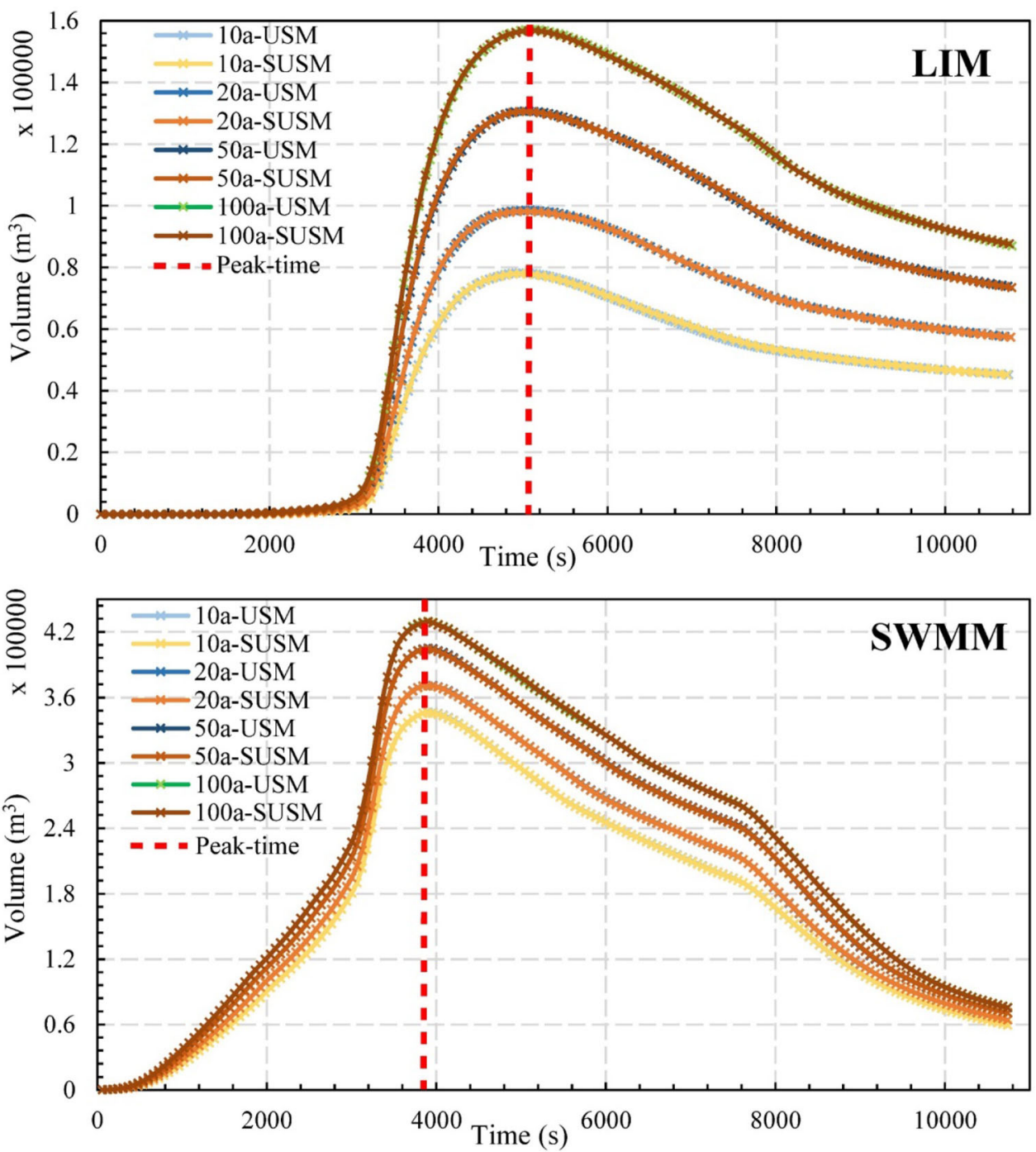
Fig. 9 The number of flooding nodes of the urban stormwater model (USM) and simplified urban stormwater model (SUSM) in the four rainfall return periods $(10 \mathrm{a}, 20 \mathrm{a}, 50 \mathrm{a}$, and 100a)

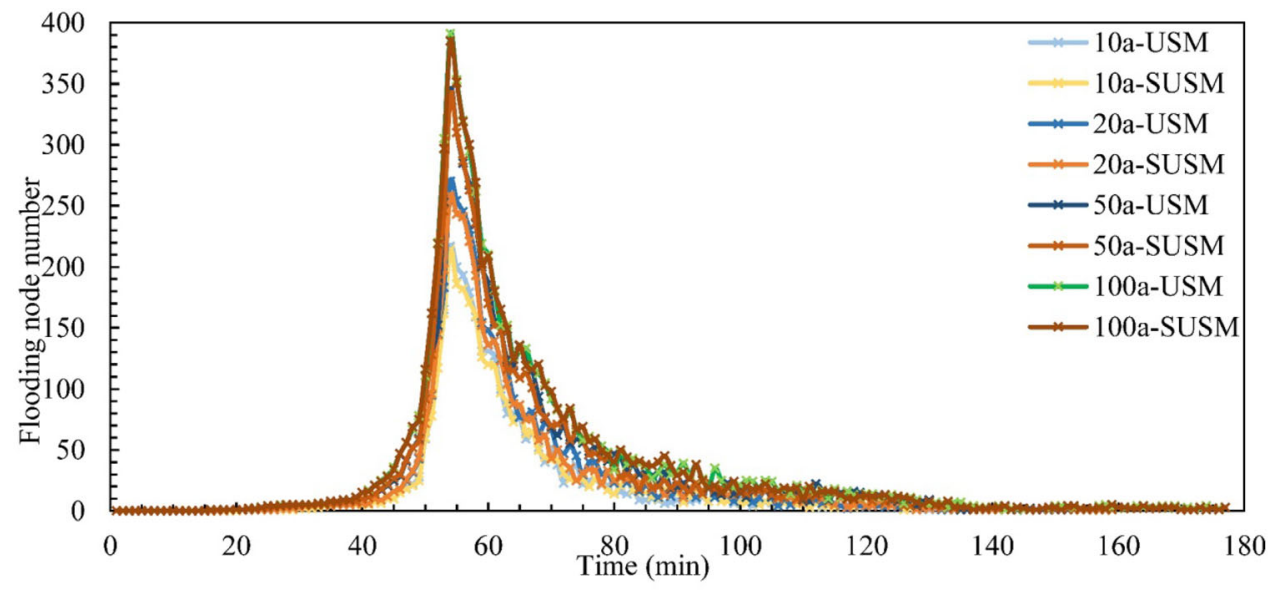

Fig. 10 Hydrographs of the outlet of the urban stormwater model (USM) and simplified urban stormwater model (SUSM) in the four rainfall return periods $(10 \mathrm{a}, 20 \mathrm{a}, 50 \mathrm{a}$, and 100a)

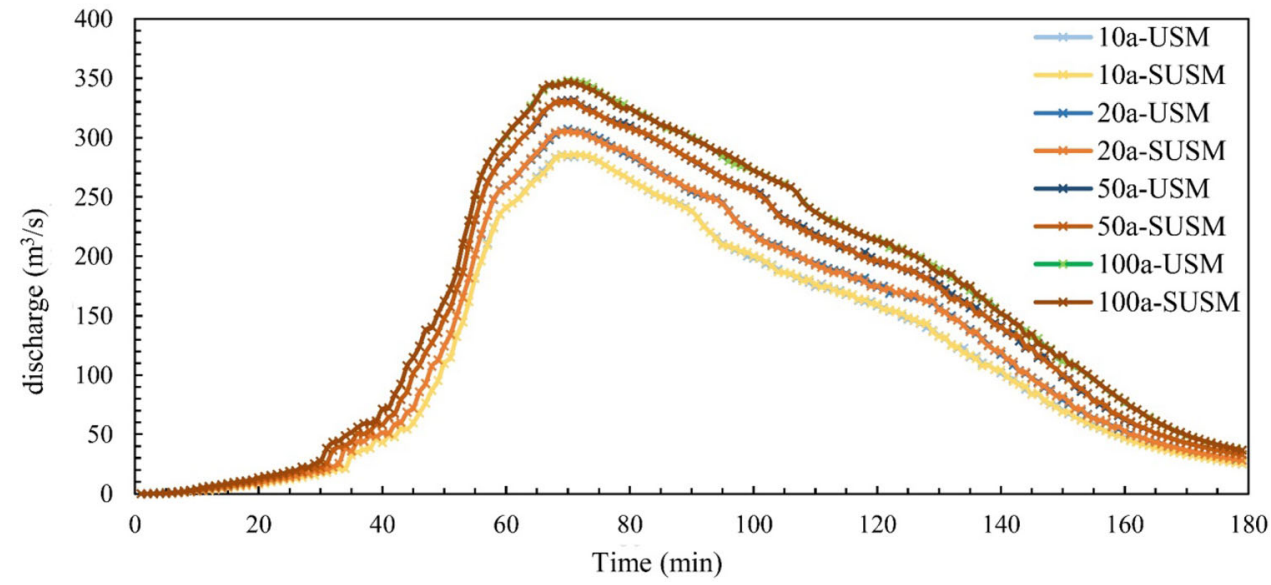

presents the water volume hydrographs of the LIM and SWMM under different scenarios.

Figure 8 reveals that the hydrographs of the two models for both the surface and the pipe system are highly consistent, and there were few differences between the two models from the perspectives of the entire flood process, total water volume, and peak water volume. However, the results modeled by the SUWM exhibited the general feature of slight underestimation $(0.2 \%-0.4 \%)$ of the peak water volume on the surface under all four rainfall scenarios. In the following discussion, this feature is reflected upon through surface submergence depth. Figure 8 also shows that the occurrence time of the peak water volume on the surface was relatively delayed (about $20 \mathrm{~min}$ ) as compared to that in the pipe network. This is because the proposed urban flood model was constructed via the combination of hydrology and hydrodynamics. The runoff generation and routing are first calculated by the SWMM hydrological model, after which the stormwater directly flows into the drainage network. The surface flow is caused by the overflow of the pipe network when the drainage capacity is insufficient.

\subsection{Flow Information of the Pipe Network}

Although the same model is used to represent the pipe network flow in the two models, there is still a certain impact on the simulated flow in the pipe network due to the different treatment methods of surface flow. Figures 9 and 10 respectively present the number of flooding nodes and the flow hydrographs of the outlets of the two models in the urban waterlogging simulation process.

From Fig. 9, it can be seen that with the occurrence of the peak rainfall, the surge process of the number of overflow nodes in the two models exhibited strong consistency. However, with the decline of the rainfall process, the number of overflow nodes presented a rapid downward trend accompanied by small fluctuation, which was due to the joint effect of the water volume of the pipe network nodes and the surface water. While both models captured this feature, they exhibited slight differences. Under the four rainfall scenarios considered in the study area, the number of maximum overflow points simulated by the SUSM was less than (in single digits) that simulated by the USM. Figure 10 reveals that the discharge processes of the outlet presented by the two models were highly consistent. 

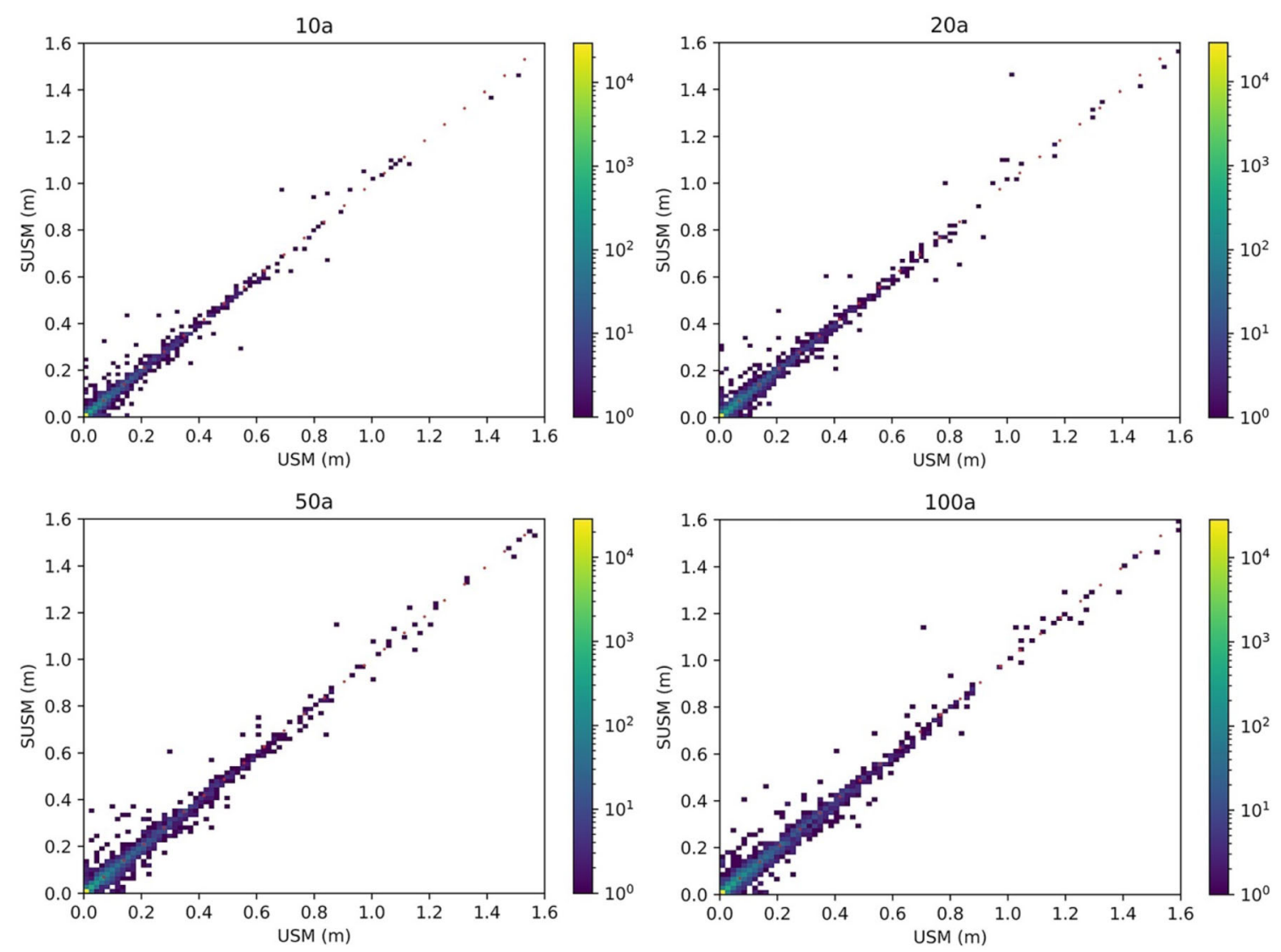

Fig. 11 2D histograms of the water depth, which present the density of raster cells in the four rainfall return periods. The $x$ - and $y$-axes represent the water depth simulated by the urban stormwater model

(USM) and simplified urban stormwater model (SUSM), respectively, and the plots are colored logarithmically

This demonstrates that due to the use of the same algorithm in the calculation of the pipe network flow and water exchange in the 1D-2D coupling, the flow characteristics of the two models are consistent in terms of the numerical simulation, even though the methods of dealing with surface water flow are different.

\subsection{Inundation Information}

The inundation information of the urban surface, especially the maximum inundation depth and maximum velocity, comprise the indicators of greatest concern in urban flood management. The simulation accuracies of the proposed SUSM model in the four rainfall return periods are presented as 2D histograms in Fig. 11 (maximum inundation depth) and Fig. 13 (maximum flow velocity).

The red diagonal dots in Fig. 11 represent the datum line with $100 \%$ accuracy, and the more accurate the model, the closer the results are to the datum line. As is evident from the figure, the 2D histograms of the maximum water depth reveal that the water depth simulation results of the two models were highly consistent. Furthermore, the intercomparison of the simulated inundation depth (obtained by

subtracting the SUSM results from the USM results, Fig. 12) shows that the USM and SUSM performed very similarly. A general overview of the maximum water depth provided by the two models reveals that the SUSM generated a very slight underestimation of the maximum water depth. Over the entire research region, the maximum water depth underestimated by the SUSM was mainly concentrated in the range of $0-0.05 \mathrm{~m}$, and the slight overestimation was made in only very few cells. Considering that the two models are based on the same computing cells with the same numerical solution scheme, these differences can be easily explained by the role played by the convective acceleration term neglected by the LIM. This illustrates that the SUSM will underestimate the water depth to a low degree when simulating the urban waterlogging process, and there may also be very few unusual differences (overestimation) with the further complication of the flow propagation process. From a quantitative perspective, the root-mean-square error (RMSE) value with respect to the USM is a key parameter for comparison, and two types of RMSE values, namely those based on global cells and wet cells, were respectively calculated. Table 4 reveals that the RMSE value of wet cells increased from 0.019 to $0.024 \mathrm{~m}$ 

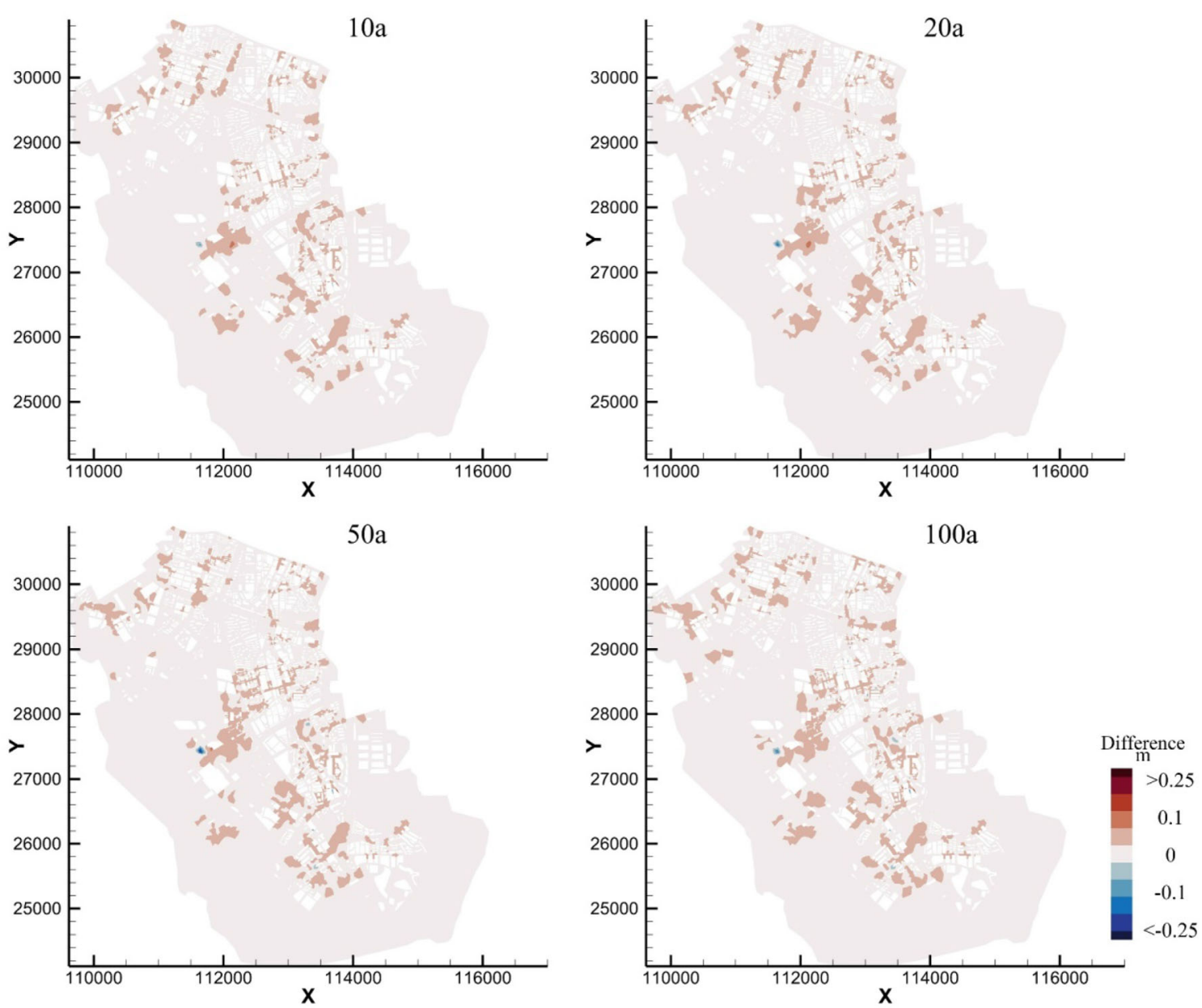

Fig. 12 Inter-comparison of the maximum inundation depth (obtained by the subtraction of the simplified urban stormwater model (SUSM) results from the urban stormwater model (USM) results)

Table 4 Root-mean-square error (RMSE) values of the water depth and flow velocity for the simplified urban stormwater model (SUSM) compared to the urban stormwater model (USM)

\begin{tabular}{llllll}
\hline RMSE & & $10 \mathrm{a}$ & $20 \mathrm{a}$ & $50 \mathrm{a}$ & $100 \mathrm{a}$ \\
\hline Global cell & Depth (m) & 0.006 & 0.007 & 0.009 & 0.009 \\
& Velocity (m/s) & 0.039 & 0.040 & 0.046 & 0.052 \\
\multirow{2}{*}{ Wet cell } & Depth (m) & 0.019 & 0.022 & 0.024 & 0.023 \\
& Velocity (m/s) & 0.128 & 0.118 & 0.131 & 0.135 \\
\hline
\end{tabular}

(and it increased from 0.006 to $0.009 \mathrm{~m}$ for global cells), and the values were always maintained at a low level.

Regarding the flow velocity, Fig. 13 reveals a regular pattern similar to that of the maximum water depth; this means that for both the water depth and flow velocity, the SUSM produced comparable results to the USM. However, the difference between them is that the dispersion of the flow-velocity histogram is significantly greater than that of the water-depth histogram; this means that compared with that of the water depth, the accuracy of the flow velocity simulated by the SUSM was slightly lower. Figure 14 presents the inter-comparison of the simulated flow velocity (obtained by the subtraction of the SUSM results from the USM results) between the two models. The figure reveals that for the entire study area, the difference between the simulation results of the two models was low, although the difference was slightly greater than that for the water depth simulation results. The SUSM also presented a low degree of underestimation in terms of the flow velocity, which was mainly concentrated in the range of $0-0.125 \mathrm{~m} / \mathrm{s}$. As is the case with the water depth, only a few cells of velocity overestimation by the SUSM were observed. In addition, the number of darker cells and blue cells was found to gradually increase with the increase of the rainfall return period. In the worst case, the maximum error was more than $0.5 \mathrm{~m} / \mathrm{s}$ for the local cells in the central area where the flooding was the most serious.

The locations of the relatively abnormal results (that is, those characterized by a slightly larger difference and 

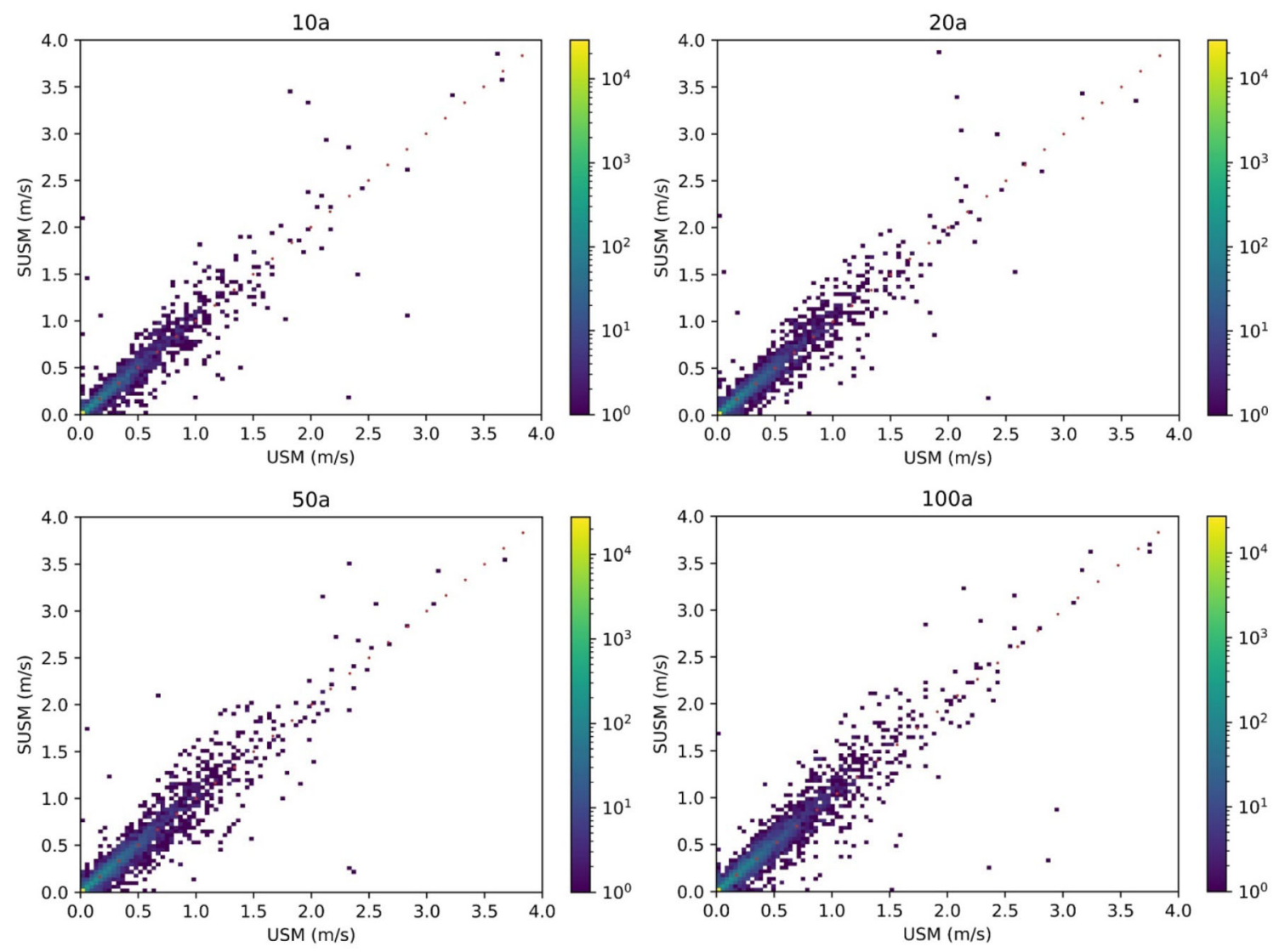

Fig. 13 2D histograms of flow velocity, which present the density of raster cells in the four rainfall return periods. The $x$ - and $y$-axes represent the flow velocities simulated by the urban stormwater model (USM) and simplified urban stormwater model (SUSM), respectively

overestimation) shown in Fig. 12 exhibited a certain degree of coincidence with those shown in Fig. 14. Moreover, the number of relatively abnormal water depth results was obviously less than the number of relatively abnormal velocity results. From these results, it can be inferred that the SUSM will underestimate the flow depth and velocity when simulating urban rainstorms, and the simulation accuracy of the velocity will be lower than that of the water depth. This can be explained by considering the effect of neglecting the convective acceleration term. In the convective acceleration term, the velocity exists in the form of a squared value, which obviously accounts for a higher proportion. Therefore, neglecting the convective acceleration term will have a greater influence on the flow velocity as compared with the flow depth. Nonetheless, the accuracy of the SUSM was found to reach a very satisfactory level.

\subsection{Froude Number Maps}

As a simplified form of the complete SWEs, there are bound to be some limitations in the applicability of the LIM to the simulation of surface water flow. de Almeida and Bates (2013) pointed out that the physical characteristics of the flow field of supercritical flow with a Froude number of greater than 1 cannot be correctly expressed by the LIM. Therefore, to discuss the limitations of the SUSM in the numerical simulation of urban stormwater, the distributions of the maximum Froude number in the research area under the four rainfall scenarios were mapped, and are shown in Fig. 15. The figure reveals that over the entire research area, the Froude number in the simulation of urban stormwater flooding was largely below 0.5 , and the value tended to increase with the increase of the rainfall return period; and the number and proportion of supercritical flow cells also increased. Table 5 reports the detailed information about the wet cells and supercritical flow cells, from which it is evident that the proportion of cells with supercritical flow was only $5.17 \%$ under the 10a rainfall scenario, and increased to $8.18 \%$ in the worst rainfall events.

For the convenience of analysis, taking the 100a rainfall event as an example, Fig. 16 presents the comparison between the distributions of the Froude number and the relative error (RE) of the maximum water depth (as compared to the USM). Table 6 shows the average RE and the 

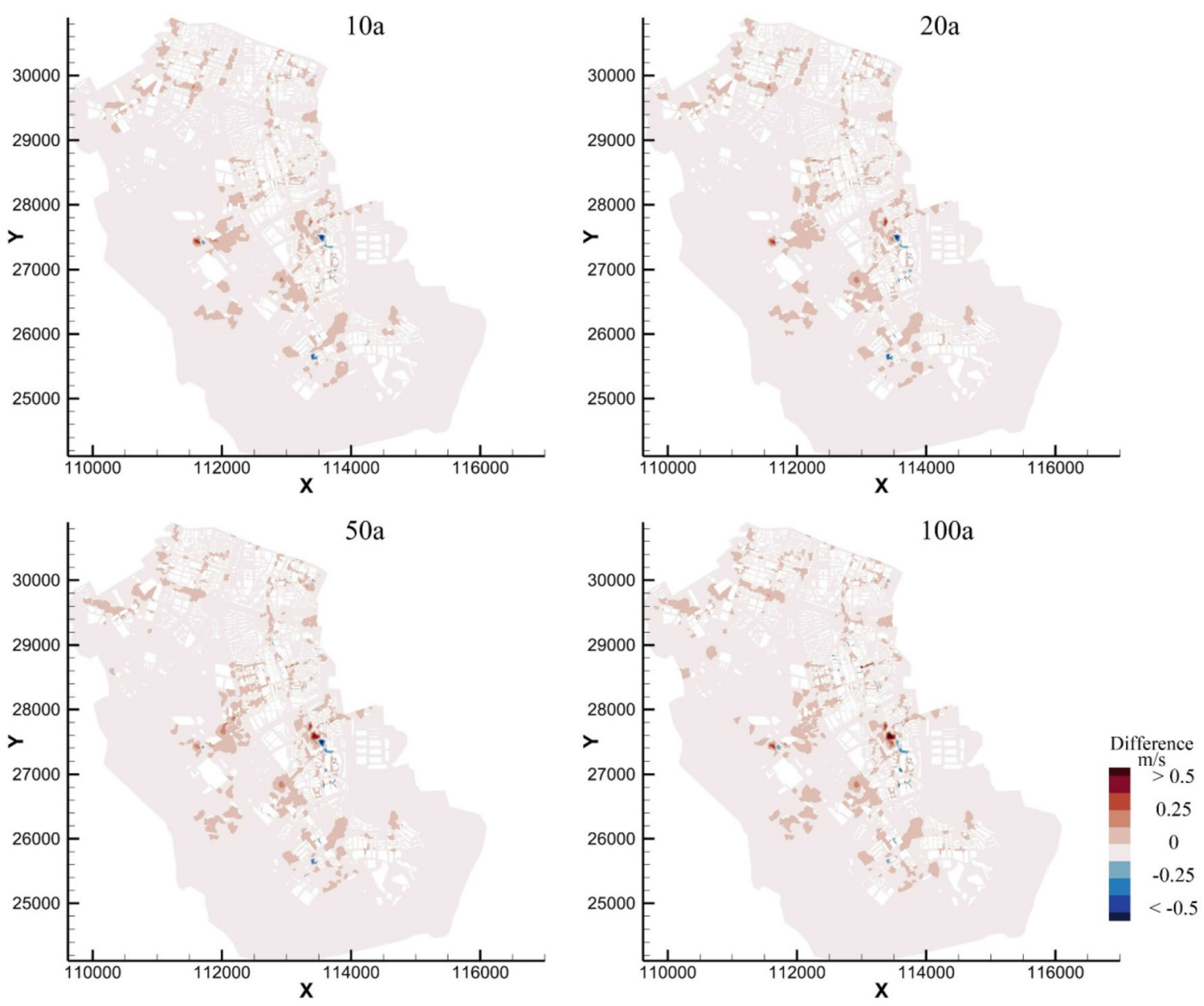

Fig. 14 Inter-comparison of the maximum flow velocity (obtained by the subtraction of the simplified urban stormwater model (SUSM) results from the urban stormwater model (USM) results)

proportion of the RE greater than $10 \%$ under different flow regimes. The range of Froude numbers from 0 to 1 was divided into three intervals: below 0.5 , between 0.5 and 1 , and above 1. From Fig. 16 and Table 6, we can find that the $\mathrm{RE}$ of the water depth exhibited a strong relationship with the Froude number intervals. When the Froude number was less than 0.5, the average RE of the maximum water depth was within $10 \%$, meaning that the accuracy of the model was very satisfactory. When the Froude number ranged from 0.5 to 1 , the accuracy of the model in some local regions declined, and there is a certain degree of increase on the average $\mathrm{RE}$ and the proportion of the RE greater than $10 \%$. However, when the flow became supercritical, the accuracy of the model exhibited some uncertainty; the average RE was generally over $20 \%$ or even as high as $100 \%$ in some local regions. In this case, the model lost its reliability. The analysis of the Froude number indicates that the proposed 1D-2D coupled urban stormwater model based on the LIM is only applicable to flow characterized by a Froude number of less than 1 . The accuracy of the model was found to be seriously affected under the supercritical flow condition because the neglect of the convective acceleration term prevents the LIM from describing the real physical flow features of the supercritical flow condition. Nevertheless, considering the proportion of supercritical flow and the calculation efficiency, the performance of the SUSM in urban stormwater simulation is still satisfactory.

\section{Conclusion}

Based on a local inertial approximate form of the SWEs in which the convective acceleration term in the momentum equation of the shallow water system is neglected, this study proposed a finite volume method with an unstructured cell-center based grid. To evaluate the performance of the LIM in urban flood modeling, the SUSM considering dual drainage system is constructed based on the LIM and SWMM. A complete SWEs-based USM was used for benchmarking. From a practical point of view, the performance and limitations of the SUSM was revealed through 

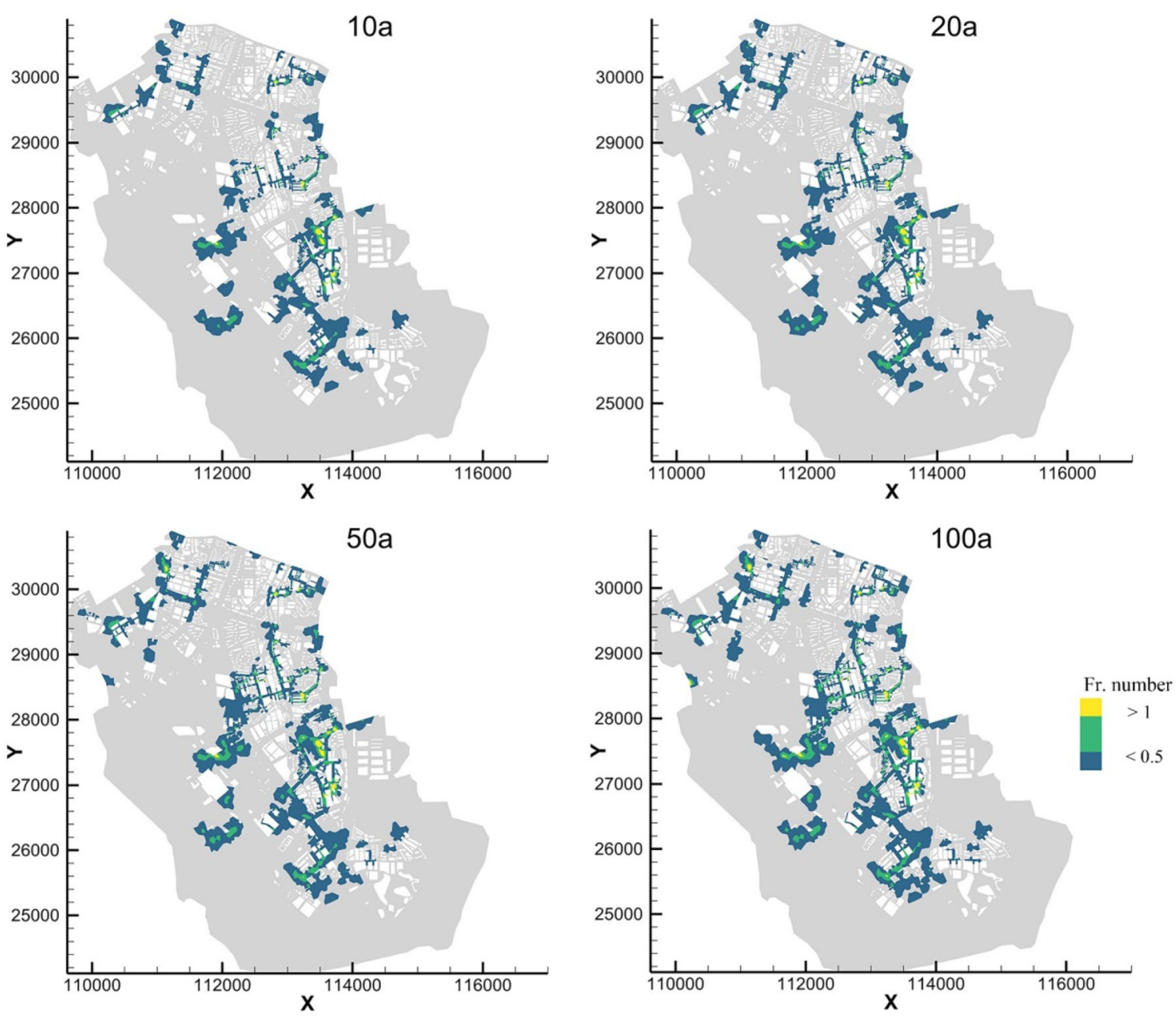

Fig. 15 Distribution of the maximum Froude number in the four return periods (10a, 20a, 50a, and 100a)

Table 5 Information on the supercritical flow cells and wet cells

\begin{tabular}{lllll}
\hline Number of Cells & $10 \mathrm{a}$ & $20 \mathrm{a}$ & $50 \mathrm{a}$ & $100 \mathrm{a}$ \\
\hline Froude number $>1$ & 137 & 199 & 307 & 380 \\
Wet & 2,645 & 3,242 & 3,878 & 4,327 \\
Proportion & $5.17 \%$ & $6.14 \%$ & $7.92 \%$ & $8.18 \%$ \\
\hline
\end{tabular}

the comparative studies in a complex urbanized region. The main conclusions are as follows.

1. The results of the benchmark tests of the surface flow model revealed that the proposed LIM solution exhibited satisfactory performance. Benefiting from the simplification of the numerical flux term and the relatively loose CFL condition, the calculation efficiency was increased by $164 \%-171 \%$ with similar accuracy as that of the SWM.

2. Through the analysis of the computational time in the practical case, it can be found that the improvement of the computational efficiency of the SUSM declined compared to the theoretical test cases. The main reason is the influence of the pipe network calculation and coupling calculation process. However, an improvement of about $140 \%$ was still achieved.

3. The numerical results of the practical urban stormwater case indicated that the SUSM and USM were in good agreement. The flow characteristics of surface water indicated that the SUSM model would slightly underestimate the depth and velocity in the entire research region, and its accuracy in describing the flow velocity is not as good as that in describing the water depth.

4. In the urban flood modeling with SUSM, the Froude number is the key factor affecting the accuracy. For the subcritical flow in urban flooding, simulation accuracy of the SUSM is satisfactory. Especially when the Froude number is less than 0.5 , the SUSM can accurately predict the flow characteristics with a relative error of less than $10 \%$. However, the neglect 

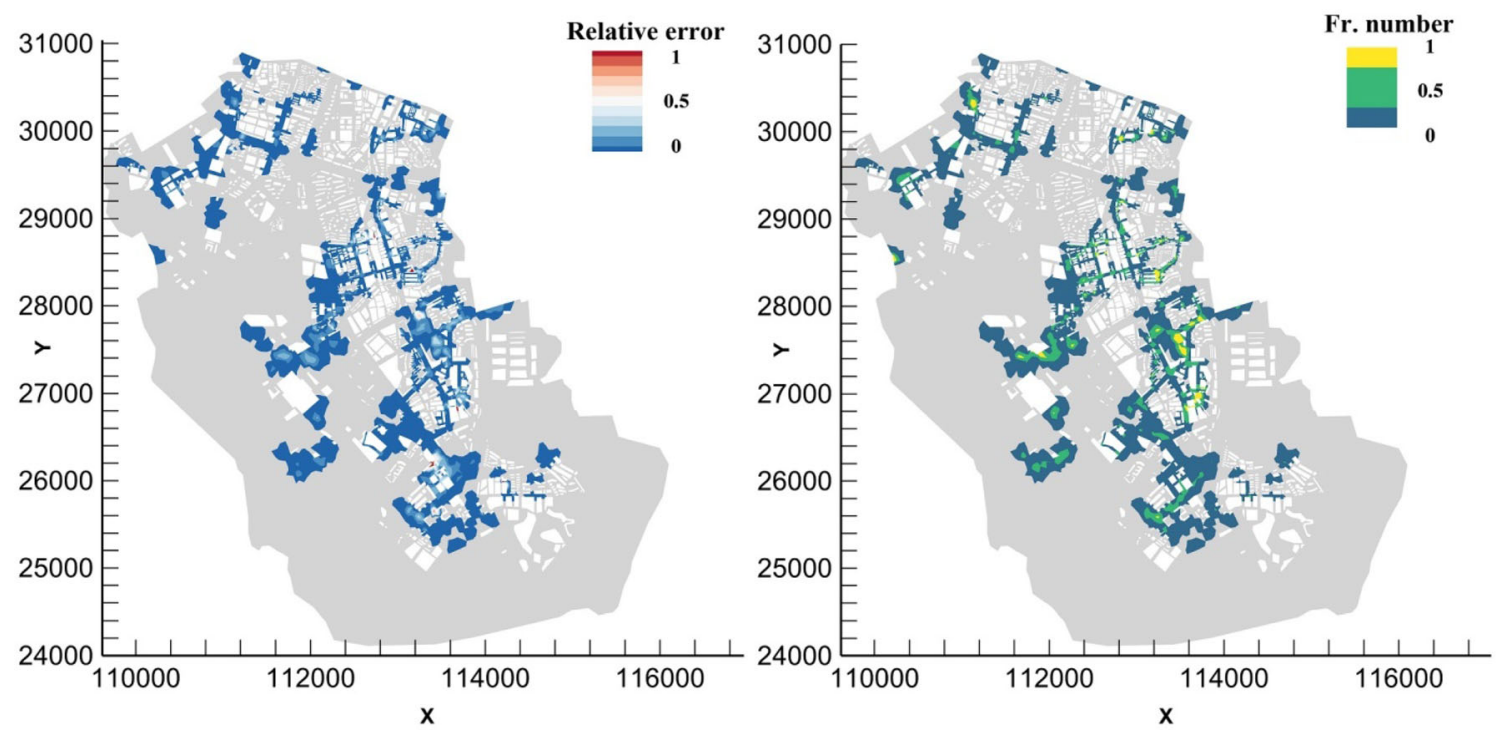

Fig. 16 Comparison of the relative errors of the maximum water depth (left) and Froude number distribution (right)

Table 6 Average relative error (RE) and the proportion of the RE greater than $10 \%$ under different flow regimes

\begin{tabular}{lll}
\hline Froude Number & Average RE & Proportion of RE $>10 \%$ \\
\hline Fr $<0.5$ & $9.74 \%$ & $18.2 \%$ \\
$0.5<\mathrm{Fr}<1$ & $11.8 \%$ & $27.3 \%$ \\
$\mathrm{Fr}>1$ & $21.3 \%$ & $59.8 \%$ \\
\hline
\end{tabular}

of the convective acceleration term prevents the SUSM from describing the real physical flow features when dealing with supercritical flow. The accuracy of the SUSM will substantially decline at localized points with a relative error of more than $100 \%$.

Models with low physical complexity can be applied to rapid flood forecasting, and can be used to formulate an evacuation plan for people to avoid flood disasters. Although this study achieved generally favorable results, it is evident that the application of the SUSM is restricted by the Froude number to a certain extent. After making a trade-off between the computational efficiency and loss of accuracy, it must be determined by the researcher whether a low physical complexity model or a high fidelity hydrodynamic model should be adopted. In addition, the combination of the two models can be a prospect of future research. For example, the SUSM may be used in a large region with a subcritical flow regime, while the USM may be used only at the localized points with a complex flow regime that require special attention.

Acknowledgements This work was supported by the National Natural Science Foundation of China (Grant Numbers 51739011 and 51879108).
Open Access This article is licensed under a Creative Commons Attribution 4.0 International License, which permits use, sharing, adaptation, distribution and reproduction in any medium or format, as long as you give appropriate credit to the original author(s) and the source, provide a link to the Creative Commons licence, and indicate if changes were made. The images or other third party material in this article are included in the article's Creative Commons licence, unless indicated otherwise in a credit line to the material. If material is not included in the article's Creative Commons licence and your intended use is not permitted by statutory regulation or exceeds the permitted use, you will need to obtain permission directly from the copyright holder. To view a copy of this licence, visit http://creativecommons. org/licenses/by/4.0/.

\section{References}

Apel, H., G.T. Aronica, H. Kreibich, and A.H. Thieken. 2009. Flood risk analyses-How detailed do we need to be?. Natural Hazards 49(1): 79-98.

Audusse, E., F. Bouchut, M.-O. Bristeau, R. Klein, and B. Perthame. 2004. A fast and stable well-balanced scheme with hydrostatic reconstruction for shallow water flows. SIAM Journal on Scientific Computing 25(6): 2050-2065.

Bates, P.D., M.S. Horritt, and T.J. Fewtrell. 2010. A simple inertial formulation of the shallow water equations for efficient twodimensional flood inundation modelling. Journal of Hydrology 387(1): 33-45.

Brufau, P., M.E. Vázquez-Cendón, and P. García-Navarro. 2002. A numerical model for the flooding and drying of irregular domains. International Journal for Numerical Methods in Fluids 39(3): 247-275.

Carsell, K.M., N.D. Pingel, and D.T. Ford. 2004. Quantifying the benefit of a flood warning system. Natural Hazards Review 5(3): 131-140.

Castro, M.J., A.M. Ferreiro Ferreiro, J.A. García-Rodríguez, J.M. González-Vida, J. Macías, C. Parés, and M.E. Vázquez-Cendón. 2005. The numerical treatment of wet/dry fronts in shallow flows: Application to one-layer and two-layer systems. Mathematical and Computer Modelling 42(3): 419-439. 
Chen, C.W., and R.P. Shubinski. 1971. Computer simulation of urban storm water runoff. Journal of the Hydraulics Division 97(2): 289-301.

Chen, A.S., M.H. Hsu, T.S. Chen, and T.J. Chang. 2005. An integrated inundation model for highly developed urban areas. Water Science and Technology 51(2): 221-229.

Chen, A.S., S. Djordjevic, J. Leandro, and D. Savic. 2007. The urban inundation model with bidirectional flow interaction between 2D overland surface and 1D sewer networks. In Proceedings of the Sixth International Conference on Sustainable Techniques and Strategies in Urban Water Management, June 2007, Lyon, France.

Chen, W., G. Huang, H. Zhang, and W. Wang. 2018. Urban inundation response to rainstorm patterns with a coupled hydrodynamic model: A case study in Haidian Island, China. Journal of Hydrology 564: 1022-1035.

Chen, W., W. Wang, G. Huang, Z. Wang, C. Lai, and Z. Yang. 2021. The capacity of grey infrastructure in urban flood management: A comprehensive analysis of grey infrastructure and the greengrey approach. International Journal of Disaster Risk Reduction 54: Article 102045.

Dazzi, S., R. Vacondio, and P. Mignosa. 2020. Internal boundary conditions for a GPU-accelerated 2D shallow water model: Implementation and applications. Advances in Water Resources 137: Article 103525.

de Almeida, G.A.M., and P. Bates. 2013. Applicability of the local inertial approximation of the shallow water equations to flood modeling. Water Resources Research 49(8): 4833-4844.

Gironás, J., L.A. Roesner, L.A. Rossman, and J. Davis. 2010. A new applications manual for the Storm Water Management Model (SWMM). Environmental Modelling \& Software 25(6): 813-814.

Hsu, M.H., S.H. Chen, and T.J. Chang. 2000. Inundation simulation for urban drainage basin with storm sewer system. Journal of Hydrology 234(1): 21-37.

Kaspersen, P.S., N.H. Ravn, K. Arnbjerg-Nielsen, H. Madsen, and M. Drews. 2017. Comparison of the impacts of urban development and climate change on exposing European cities to pluvial flooding. Hydrology and Earth System Sciences 21(8): 4131-4147.

Leandro, J., A.S. Chen, S. Djordjević, and D.A. Savić. 2009. Comparison of 1D/1D and 1D/2D coupled (sewer/surface) hydraulic models for urban flood simulation. Journal of Hydraulic Engineering 135(6): 495-504.

Leandro, J., P. Lopes, R. Carvalho, P. Páscoa, R. Martins, and M. Romagnoli. 2014. Numerical and experimental characterization of the 2D vertical average-velocity plane at the center-profile and qualitative air entrainment inside a gully for drainage and reverse flow. Computers \& Fluids 102: 52-61.

Liang, Q., X. Xia, and J. Hou. 2015. Efficient urban flood simulation using a GPU-accelerated SPH model. Environmental Earth Sciences 74(11): 7285-7294.

Martins, R., J. Leandro, and S. Djordjević. 2015. A well balanced Roe scheme for the local inertial equations with an unstructured mesh. Advances in Water Resources 83: 351-363.

McCallum, I., W. Liu, L. See, R. Mechler, A. Keating, S. HochrainerStigler, J. Mochizuki, and S. Fritz et al. 2016. Technologies to support community flood disaster risk reduction. International Journal of Disaster Risk Science 7(2): 198-204.

Neal, J., I. Villanueva, N. Wright, T. Willis, T. Fewtrell, and P. Bates. 2012. How much physical complexity is needed to model flood inundation?. Hydrological Processes 26(15): 2264-2282.

Néelz, S., and G. Pender. 2013. Benchmarking the latest generation of 2D hydraulic modelling packages. Bristol, UK: Environment Agency.

Ponce, V.M. 1990. Generalized diffusion wave equation with inertial effects. Water Resources Research 26(5): 1099-1101.

Roe, P.L. 1981. Approximate Riemann solvers, parameter vectors, and difference schemes. Journal of Computational Physics 43(2): 357-372.

Rossman, L.A., and W. Huber. 2017. Storm Water Management Model reference manual volume II-Hydraulics. Washington, DC: US Environmental Protection Agency.

Santos, P.P., and E. Reis. 2018. Assessment of stream flood susceptibility: A cross-analysis between model results and flood losses. Journal of Flood Risk Management 11(S2): S1038S1050.

Xia, X., and Q. Liang. 2018. A new efficient implicit scheme for discretising the stiff friction terms in the shallow water equations. Advances in Water Resources 117: 87-97.

Xia, X., Q. Liang, X. Ming, and J. Hou. 2017. An efficient and stable hydrodynamic model with novel source term discretization schemes for overland flow and flood simulations. Water Resources Research 53(5): 3730-3759.

Xia, X., Q. Liang, and X. Ming. 2019. A full-scale fluvial flood modelling framework based on a high-performance integrated hydrodynamic modelling system (HiPIMS). Advances in Water Resources 132: Article 103392.

Yang, Y., L. Sun, R. Li, J. Yin, and D. Yu. 2020. Linking a Storm Water Management Model to a novel two-dimensional model for urban pluvial flood modeling. International Journal of Disaster Risk Science 11(4): 508-518. 